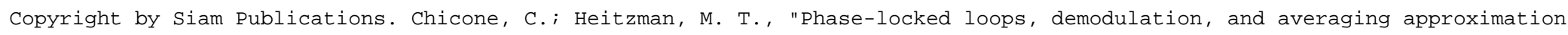
time-scale extensions," SIAM J. Appl. Dyn. Syst., 12(2), 674-721, (2013); DOI: 10.1137/120894646

\title{
Phase-Locked Loops, Demodulation, and Averaging Approximation Time-Scale Extensions*
}

\author{
Carmen Chicone $^{\dagger}$ and Michael T. Heitzman ${ }^{\ddagger}$
}

\begin{abstract}
Among the many applications of the phase-locked loop (PLL), a device used extensively in telecommunications and electronics, is the demodulation of modulated carrier signals. The PLL contains a voltage controlled oscillator (VCO) that tracks a reference signal whose frequency may be changing. This is accomplished through a feedback mechanism - the VCO's frequency is adjusted by a control signal that, after filtering, depends mostly on the phase difference between the reference and VCO output. Phase-lock describes an operating state for which this phase difference remains constant. During nearly phase-locked operation, the filtered signal controlling the VCO approximates the demodulation of the reference. A standard model is used to give a rigorous mathematical explanation of the described operation of the PLL in a physically realistic operating regime. While the model does not allow strict phase-locking, a theorem is formulated and proved that predicts operation near an attracting torus with quasi-periodic flow in the state space. The proof uses high-order averaging, a new result on extension of the averaging estimate to the forward infinite time-scale, and continuation theory for invariant manifolds. For the averaged system (equivalent to a simplified model that assumes ideal filtering), we obtain an approximation for solutions on an attracting invariant torus (for quasi-periodic reference signal modulation of sufficiently small amplitude and frequency), in which the dominant response of the filtered control signal is the demodulation of the reference signal, up to a rescaling and constant shift. Furthermore, we show that the full model (allowing nonideal filtering) also has an attracting torus, on which solutions exhibit the same dominant response. In addition, some results on continuation of invariant manifolds, which may have applications beyond the PLL, are proved.
\end{abstract}

Key words. phase-locked loop, demodulation, averaging, invariant manifold, time-scale extension

AMS subject classifications. $34 \mathrm{C} 29,34 \mathrm{C} 45$

DOI. $10.1137 / 120894646$

1. Introduction. The phase-locked loop (PLL) is a ubiquitous device widely used in telecommunications, signal processing, and computer electronics for applications ranging from demodulation, extracting messages in signals with background noise, and frequency synthesis to distributing clock timing pulses in microprocessors. It is designed as a feedback loop comprised of an oscillator whose instantaneous frequency is modulated by a control signal formed from a (low-pass) filtered combination of the oscillator output and a reference signal. In case the reference and oscillator output signals are (phase or frequency) modulated sinusoids and are combined by multiplication, their product can be written as a sum of sinusoids whose arguments are the sum and difference of the respective phases of the reference and oscillator output signals. In this case, whenever the instantaneous frequencies of the reference and os-

\footnotetext{
${ }^{*}$ Received by the editors October 10, 2012; accepted for publication (in revised form) by T. Sauer February 25, 2013; published electronically May 2, 2013.

http://www.siam.org/journals/siads/12-2/89464.html

${ }^{\dagger}$ Department of Mathematics, University of Missouri, Columbia, MO 65201 (chiconec@missouri.edu).

${ }^{\ddagger}$ Department of Mathematics, Virginia Tech, Blacksburg, VA 24061 (heitzman@math.vt.edu).
}

674 
cillator output signals remain near a constant carrier frequency (i.e., the modulation is small), the sum term will be mostly blocked by the low-pass filter, the difference term will pass relatively unattenuated, and the dominant control signal modulating the oscillator's frequency will depend on the phase difference between the reference and oscillator output. Ideally, this feedback mechanism causes the oscillator output to track the reference signal.

While much excellent work on modeling and applications of PLLs is available, most models in the PLL literature are derived from the physical argument given above: small modulation frequency (in comparison to the constant carrier frequency) and presumed ideal low-pass filter behavior are used as justification for assuming that the control signal modulating the oscillator's instantaneous frequency depends only on the phase difference between the reference signal and the oscillator output (see, for example, [1], [15], [11], [21], [4], [2], [8], [5]). The resulting traditional model takes the form of a damped, driven pendulum-type ODE for the phase difference, in which time dependent driving arises from modulation of the reference signal. (In case the reference signal is unmodulated, the driving reduces to a constant torque proportional to the difference between the free running oscillator frequency and the carrier frequency of the reference.)

Notable exceptions are [16], [12], and [6], which present full models retaining the high frequency sum term arising from the product of the reference and oscillator output signals, so that after (nonideal) low-pass filtering, the control signal depends on both the difference and, to a lesser extent, the sum (sometimes called the ripple) of the reference and oscillator output phases. The PLL model in [6] is limited to reference signals that are pure sinusoids with no modulation.

In [16], a combination of averaging and multiple time-scale techniques is used to obtain a simplified model in which the high frequency sum term has been eliminated (although the ripple amplitude persists due to the particular averaging technique and generalized model of the oscillator used). A detailed qualitative analysis of the averaged system is performed - in the case of no modulation or time independent modulation of the reference signal, linearized stability of rest points is determined, and the conditions for their existence are used to ascertain hold and pull-in frequency intervals for the PLL, which are then extended in a somewhat dubious manner to the case of time dependent modulation. No mathematically rigorous estimates are given for how closely or on which time-scale the averaged system approximates the full system; rather, numerical simulations are presented in an attempt to justify the approximation.

A more mathematical treatment is provided by [12], including analysis of both the averaged and full systems and a more general exposition of dynamics on the torus. However, for the most part, the PLL application in [12] is restricted to the case of an unmodulated reference signal. One brief example involving modulation of the reference is presented, but only for the averaged system where the phase sum term has been dropped. Furthermore, analysis of the full system in [12] relies heavily on perturbation of an artificial small parameter placed arbitrarily as a coefficient of the phase sum term, but not the phase difference term. (This artificial small parameter should not be confused with the small parameter $\epsilon$ that allows the averaging procedure described below; the parameter $\epsilon$ below arises from physical quantities and appears as a coefficient in both the phase sum and phase difference terms.)

Copyright (c) by SIAM. Unauthorized reproduction of this article is prohibited. 
The full models in both [16] and [12] take the form of a nonautonomous ODE,

$$
\dot{x}=\epsilon F(x, t, \epsilon),
$$

in which $x$ is a slowly varying state vector due to the small parameter $\epsilon$. The averaged system is then given by the autonomous ODE,

$$
\dot{x}=\epsilon \bar{F}(x, \epsilon),
$$

where

$$
\bar{F}(x, \epsilon):=\lim _{T \rightarrow+\infty} \frac{1}{T} \int_{0}^{T} F(x, t, \epsilon) d t .
$$

A standard result in averaging theory states that, with appropriate conditions on $F$, solutions of (1.2) approximate solutions of (1.1) to order $\epsilon$ on a time-scale of order $1 / \epsilon$ (see [19], for example).

Our approach differs from both [16] and [12], with a focus on the PLL application to demodulation and a mathematically rigorous time extension of the approximation by an averaged system to the forward infinite time-scale. Similar to [16] and [12], we develop a full PLL model that retains the phase sum term arising from the product of the reference and oscillator output signals. Our full model can be expressed in the action-angle form

$$
\begin{aligned}
& \dot{x}=\sum_{p=K}^{N} \epsilon^{p} f_{p}(x, \theta, \epsilon), \\
& \dot{\theta}=\Omega+O(\epsilon),
\end{aligned}
$$

where the fast angle $\theta \in \mathbb{T}^{1}$ (the one-dimensional torus) denotes the sum of the reference and oscillator output phases, $K$ and $N$ are positive integers, $\Omega$ is a positive constant representing twice the carrier frequency, and $\epsilon$ is a small positive parameter (various powers of which represent the small ratio of modulation frequency to carrier frequency, small modulation amplitude, and small ratio of the low-pass filter inverse time constant to the carrier frequency). The components of the slow action vector $x$ include the low-pass filter output, the reference and oscillator output phase difference, and the reference signal modulation phase.

A rigorous method of averaging for action-angle systems of the form (1.4) can be described as follows (see [19] for an excellent modern reference on averaging theory). There exists a smooth, near-identity transformation of the form $I+O\left(\epsilon^{K}\right), 2 \pi$-periodic in $\theta$, which transforms the full system (1.4) into the equivalent system

$$
\begin{aligned}
& \dot{x}=\sum_{p=K}^{N} \epsilon^{p} \tilde{f}_{p}(x, \epsilon)+O\left(\epsilon^{N+1}\right), \\
& \dot{\theta}=\Omega+O(\epsilon),
\end{aligned}
$$

where the dependence in the (slow) action vector field on the (fast) angle $\theta$ has been pushed to order $\epsilon^{N+1}$. Truncation of system (1.5) to order $\epsilon^{N}$ decouples the action vector $x$ from the 
angle $\theta$, yielding the $(N$ th-order $)$ averaged system

$$
\dot{x}=\sum_{p=K}^{N} \epsilon^{p} \tilde{f}_{p}(x, \epsilon),
$$

which for our particular choice of $\epsilon$ scaling (see (5.4)) corresponds exactly to the simplified system of the traditional PLL model, obtained by discarding the high frequency phase sum term due to physical reasoning.

Gronwall's inequality can be used to show that solutions of the averaged system (1.6) approximate the action component of solutions of the transformed-not-truncated system (1.5) to order $\epsilon^{N-K+1}$ on a time-scale of $1 / \epsilon^{K}$, which in turn approximates the action component of solutions of the full system (1.4) to order $\epsilon^{K}$. Thus, for $K \leq N-K+1$, solutions of the averaged system (1.6) approximate the action components of solutions of the original full system (1.4) to order $\epsilon^{K}$ on a time-scale of $1 / \epsilon^{K}$ (for our model, $K=2$ and $N=6$ ). This is a standard result of higher-order averaging.

We extend this standard result to the forward infinite time-scale by employing a technique introduced by Sanchez-Palencia in [18] and Sanders in [20]. They use contraction in the flow of the action variable of the truncated system to compensate for the exponential growth terms that arise in the standard Gronwall estimate of the difference between the solutions of the truncated and not truncated systems. In [20], Sanders obtains the desired forward infinite time extension of the approximation of the averaged system to the not truncated system in case the averaged system has a hyperbolic attracting rest point (corresponding to an attracting limit cycle in the action-angle system). For our PLL model, in case the modulation of the reference signal is quasi-periodic and $\epsilon$ is sufficiently small, the averaged system (1.6) has a hyperbolic attracting torus rather than a rest point. Thus, Sanders' result cannot be directly applied. Fortunately, the basic techniques used by Sanders can be modified to our flow with an attracting torus.

As previously mentioned, the main application of the PLL we consider is in demodulation. For the averaged system (1.6) (corresponding to the traditional simplified PLL model), we obtain an approximate formula for solutions on the attracting torus (for quasi-periodic reference signal modulation and sufficiently small $\epsilon$ ), in which the dominant response of the low-pass filter output is the demodulation of the reference signal, up to a rescaling and constant shift. Additionally, we show that the full system (1.4) also has an attracting torus, on which solutions exhibit the same dominant response (asymptotically in time). Furthermore, the near-identity transformation provides high frequency correction terms that restore some of the high frequency content lost in the averaging process, allowing for an improved approximation. These correction terms could have direct application in PLL design for demodulation, suggesting a value for the total loop gain that will likely minimize distortion of the demodulation of the reference signal appearing in the low-pass filter output.

The paper is organized as follows. Section 2 introduces the model differential equations for the PLL used in demodulation circuits. Section 3 contains a statement and analysis of the simplified model that is most often encountered in the engineering literature, and section 4 contains a discussion of the most basic features of the full model. In particular, for the simplified model, strict phase-lock (constant phase difference between the reference and

Copyright (c) by SIAM. Unauthorized reproduction of this article is prohibited. 


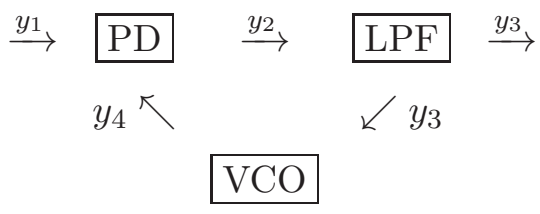

Figure 1. PLL feedback loop.

oscillator output) is possible only in the case of no modulation (for which "hold" and "pull-in" frequency intervals are traditionally obtained), whereas in the full model strict phase-lock is not possible at all. In section 5 the full model is recast in dimensionless form, and small parameters, which are physically realistic, are identified. High-order averaging of the full model is performed and discussed in section 6 , and estimates of the difference between solutions of the full and averaged systems are provided on the forward infinite time-scale. Continuation theory for invariant manifolds is used in section 7 to determine approximate solutions of the averaged system on an attracting torus. In section 8, the approximate solutions for the averaged system, combined with the averaging estimates and transformation from section 6 , are used to obtain approximate solutions for the full system on its attracting torus (whose existence is again obtained by using continuation theory).

The theorem stating that the averaging estimates can be extended to the infinite time-scale is proved in Appendix A. Appendix B states theorems on the continuation and attraction of invariant manifolds in case the unperturbed torus consists of rest points or supports a translation flow. While more general results have been proved by Hale [10], Hirsch, Pugh, and Shub [13], and Fenichel [7] for the normally hyperbolic case, the special cases of those results employed in this paper are more easily and efficiently obtained with proofs, given in Appendix B, that are based on the implicit function theorem. In Appendix C, a continuation theory for invariant manifolds is developed and applied to a class of systems (action-angle form with several angles) that are more general than the specific equations used to model the PLL. In these latter appendices we formulate and prove new continuation results for non-normally hyperbolic invariant manifolds. These results should have applications beyond the PLL.

2. PLL governing equations. In telecommunications, messages are often transmitted by encoding them as modulations of the phase or frequency of carrier signals. The process of extracting the modulation of a carrier signal (and hence reconstructing the message) is called demodulation, a task which is often accomplished by PLLs.

A (phase or frequency) modulated sinusoid can be represented by a function of the form

$$
t \mapsto A \sin \theta(t),
$$

where the real number $A$ is the amplitude and the real-valued function $t \mapsto \theta(t)$ gives the phase at time $t$. The time derivative of the phase, $\dot{\theta}(t)$, is called the instantaneous (angular) frequency. In case $\theta(t)=\omega t+\alpha(t)$ for some $\omega>0$ and real-valued function $\alpha$, the function $\alpha$ can be viewed as a modulation of the phase, and its time derivative, $\dot{\alpha}$, can be viewed as a modulation of the constant frequency $\omega$.

A PLL consists of three main components: a phase detector (PD), low-pass filter (LPF), and voltage controlled oscillator (VCO). In Figure 1, the input (or reference) signal, $y_{1}$, and 
the output of the VCO, $y_{4}$, are modulated sinusoids that are combined in the PD (often a multiplier mixer). The PD output, $y_{2}$, depends on the phase difference between $y_{1}$ and $y_{4}$, among other things. This is fed through the LPF, which produces $y_{3}$, an improved measure of the phase difference between $y_{1}$ and $y_{4}$ (under appropriate conditions). The instantaneous operating frequency of the VCO is governed by $y_{3}$, so that ideally the frequency of $y_{4}$ is shifted closer to the frequency of $y_{1}$. If $y_{1}$ and $y_{4}$ maintain exactly the same instantaneous frequency, their phase difference is constant, and they are said to be in phase-lock. Assuming that the instantaneous VCO frequency is a linear function of $y_{3}$, then during phase-lock, $y_{3}$ would be the demodulation of the modulated input $y_{1}$ (up to additive and multiplicative constants). Thus, when used as a demodulator, the LPF output, $y_{3}$, is the output of the PLL device. A more mathematically precise description proceeds as follows.

Suppose that the input and VCO output signals have the form

$$
\begin{aligned}
& y_{1}(t)=f\left(\theta_{\text {in }}(t)\right), \\
& y_{4}(t)=g(\theta(t)),
\end{aligned}
$$

where $f$ (respectively, $g$ ) is a $2 \pi$-periodic function of the input phase $\theta_{\text {in }}$ (respectively, the VCO output phase $\theta$ ). Treating the PD as a multiplier mixer, the PD output $y_{2}$ is given by

$$
\begin{aligned}
y_{2}(t) & =K_{p} y_{1}(t) y_{4}(t) \\
& =K_{p} f\left(\theta_{\text {in }}(t)\right) g(\theta(t)),
\end{aligned}
$$

where $K_{p}$ is a positive constant, not to be confused with the PD sensitivity, from which it differs by factors of $1 / 2$ and the amplitudes of $y_{1}$ and $y_{2}$ (see (2.14)).

In case the LPF is a lead-lag filter (an RC filter commonly used in PLL applications), the relationship between $y_{2}$ and $y_{3}$ is given by the following ODE:

$$
y_{3}+\tau_{1} \dot{y}_{3}=y_{2}+\tau_{2} \dot{y}_{2},
$$

where $\tau_{1}$ and $\tau_{2}$ are nonnegative constants. In most applications, $\tau_{2} \ll \tau_{1}$ (see [21, p. 110]). For simplicity, we treat the case $\tau_{2}=0$, in which the LPF reduces to a lag filter and $(2.4)$ reduces to the ODE

$$
y_{3}+\tau_{1} \dot{y}_{3}=y_{2} .
$$

We assume that the instantaneous frequency of the VCO output is controlled by $y_{3}$ through the linear relationship

$$
\dot{\theta}=\omega_{0}+K_{v} y_{3},
$$

where the positive constant $\omega_{0}$ is called the free-running VCO angular frequency and $K_{v}$ is a positive constant called the VCO sensitivity.

Equations (2.1)-(2.3) combined with (2.5)-(2.6) yield the coupled ODE system

$$
\begin{aligned}
\dot{y}_{3} & =-\frac{1}{\tau_{1}} y_{3}+\frac{K_{p}}{\tau_{1}} f\left(\theta_{\text {in }}\right) g(\theta), \\
\dot{\theta} & =\omega_{0}+K_{v} y_{3}
\end{aligned}
$$

Copyright $\odot$ by SIAM. Unauthorized reproduction of this article is prohibited. 
for the LPF voltage output, $y_{3}$, and the VCO output phase, $\theta$. Alternatively, $\theta$ may be eliminated from the system by integration, yielding the single integro-differential equation

$$
y_{3}+\tau_{1} \dot{y}_{3}=K_{p} f\left(\theta_{\mathrm{in}}\right) g\left(\omega_{0} t+K_{v} \int_{0}^{t} y_{3}(s) d s\right)
$$

where (without loss of generality) we have assumed that $\theta(0)=0$.

One more alternative is to eliminate $y_{3}$ from (2.7), yielding the second-order ODE

$$
\tau_{1} \ddot{\theta}+\dot{\theta}-K_{v} K_{p} f\left(\theta_{\text {in }}(t)\right) g(\theta)=\omega_{0} .
$$

In case the function $g$ is sinusoidal, (2.9) describes a damped pendulum driven by parametric forcing plus a constant torque.

By changing variables to the phase difference

$$
\phi:=\theta_{\text {in }}-\theta,
$$

system (2.7) is recast as

$$
\begin{aligned}
\dot{\phi} & =-K_{v} y_{3}+\dot{\theta}_{\text {in }}-\omega_{0}, \\
\dot{y}_{3} & =-\frac{1}{\tau_{1}} y_{3}+\frac{K_{p}}{\tau_{1}} f\left(\theta_{\text {in }}\right) g\left(\theta_{\text {in }}-\phi\right),
\end{aligned}
$$

and (2.9) becomes

$$
\tau_{1} \ddot{\phi}+\dot{\phi}+K_{v} K_{p} f\left(\theta_{\text {in }}\right) g\left(\theta_{\text {in }}-\phi\right)=\tau_{1} \ddot{\theta}_{\text {in }}+\dot{\theta}_{\text {in }}-\omega_{0} .
$$

Let $f$ and $g$ be sinusoids given by

$$
f(\theta)=A_{f} \sin \theta, \quad g(\theta)=A_{g} \cos \theta,
$$

where $A_{f}$ and $A_{g}$ are positive constants (the amplitudes of $y_{1}$ and $y_{4}$ ). The PD output, $y_{2}$, can then be written as

$$
\begin{aligned}
y_{2} & =K_{p} f\left(\theta_{\text {in }}\right) g\left(\theta_{\text {in }}-\phi\right) \\
& =K_{p} A_{f} A_{g} \sin \theta_{\text {in }} \cos \left(\theta_{\text {in }}-\phi\right) \\
& =K_{d}\left(\sin \phi+\sin \left(2 \theta_{\text {in }}-\phi\right)\right),
\end{aligned}
$$

where $K_{d}:=K_{p} A_{f} A_{g} / 2$ represents the phase detector sensitivity. Recall from (2.10) that $\phi$ is the phase difference between the input and VCO output signals; thus $2 \theta_{\text {in }}-\phi$ is their phase sum.

In this case, (2.11) and (2.12) are transformed into the system

$$
\begin{aligned}
\dot{\phi} & =-K_{v} y_{3}+\dot{\theta}_{\text {in }}-\omega_{0}, \\
K_{v} \dot{y}_{3} & =-\frac{K_{v}}{\tau_{1}} y_{3}+\frac{K_{0}}{\tau_{1}}\left(\sin \phi+\sin \left(2 \theta_{\text {in }}-\phi\right)\right)
\end{aligned}
$$

Copyright $($ ) by SIAM. Unauthorized reproduction of this article is prohibited. 
and the second-order differential equation

$$
\tau_{1} \ddot{\phi}+\dot{\phi}+K_{0} \sin \phi+K_{0} \sin \left(2 \theta_{\text {in }}-\phi\right)=\tau_{1} \ddot{\theta}_{\text {in }}+\dot{\theta}_{\text {in }}-\omega_{0},
$$

respectively, where $K_{0}:=K_{d} K_{v}$ is called the total loop gain. In a simplified model that discards the term $K_{0} \sin \left(2 \theta_{\text {in }}-\phi\right)$ (due to filtering or averaging, as described in the next section), and assuming steady state operation for small phase difference $\phi$ and constant $\dot{\theta}_{\text {in }}$, the differential equation reduces to the statement

$$
\dot{\theta}_{\text {in }}-\omega_{0}=K_{0} \phi
$$

In other words, the phase difference between the input and the free running VCO is linearly related to their frequency difference by $K_{0}$.

3. Simplified model. The traditional simplified PLL model found extensively in the engineering literature (see [1], [15], [11], [21], [4], [2], [8], [5], for example) is derived by assuming that the modulation frequency is small compared to the carrier frequency, and that the LPF behaves as an ideal LPF. Recall that $y_{2}$, the PD output (given by (2.14)), is fed into the LPF. In many applications, the input $y_{1}$ is a modulated signal whose instantaneous frequency, $\dot{\theta}_{\text {in }}$, is near the carrier frequency, $\omega_{c} \gg 1$. During phase-locked operation, the phase difference $\phi$ is constant. Thus, if the PLL operates in the nearly phase-locked regime (i.e., $\dot{\phi} \approx 0$ ), then $\sin \left(2 \theta_{\text {in }}-\phi\right)$ is a high frequency term (with instantaneous frequency $2 \dot{\theta}_{\text {in }}-\dot{\phi} \approx 2 \omega_{c}$ ) that will be mostly blocked by the LPF, whereas $\sin \phi$ is a low frequency term (with instantaneous frequency $\dot{\phi} \approx 0$ ) that will pass through the LPF mostly unattenuated. Based on this physical reasoning, the term containing $\sin \left(2 \theta_{\text {in }}-\phi\right)$ is dropped from (2.15) and (2.16) to obtain the simplified system

$$
\begin{aligned}
\dot{\phi} & =-K_{v} y_{3}+\dot{\theta}_{\text {in }}-\omega_{0}, \\
K_{v} \dot{y}_{3} & =-\frac{K_{v}}{\tau_{1}} y_{3}+\frac{K_{0}}{\tau_{1}} \sin \phi
\end{aligned}
$$

and the simplified second-order ODE

$$
\tau_{1} \ddot{\phi}+\dot{\phi}+K_{0} \sin \phi=\tau_{1} \ddot{\theta}_{\text {in }}+\dot{\theta}_{\text {in }}-\omega_{0},
$$

respectively. These ODEs also model the motion of a damped, driven pendulum.

The physical argument leading from the full equations (2.15) or (2.16) to the simplified equations (3.1) or (3.2) can be understood in a mathematical sense using the theory of averaging. Heuristically, $\phi$ is a slow angle compared to the fast angle $\theta_{\text {in }}$. Naively averaging over the fast angle $\theta_{\text {in }}$ in (2.15) and (2.16) (while retaining $\dot{\theta}_{\text {in }}$ as a known function) yields (3.1) and (3.2). In other words, on a time interval over which $\phi$ remains essentially constant (compared to $\theta_{\text {in }}$, which in the same time interval traverses many cycles), (2.16) and (3.2) should exhibit similar dynamics. In the following sections, we make this naive averaging argument mathematically rigorous by employing an averaging transformation and obtaining an appropriate estimate for the difference between the solutions of the averaged and not averaged equations. Furthermore, using techniques from [20], we extend the time interval of the validity of the 
averaging approximation by taking advantage of the existence of an attractor in the averaged ODEs.

First, we continue analysis of the simplified ODE (3.2) (or the equivalent system (3.1)) in relation to the physical operation of the PLL. In case the input signal $y_{1}$ is phase- or frequency modulated, we may write

$$
\theta_{\text {in }}(t)=\omega_{c} t+\alpha(t)
$$

where $\omega_{c}$ is the constant carrier angular frequency and $\alpha(t)$ is the modulation of the phase. For phase modulation (PM), the message would be $\alpha(t)$, whereas for frequency modulation (FM), the message would be $\dot{\alpha}(t)$. During phase-lock, we have $\dot{\theta}_{\text {in }} \equiv \dot{\theta}$. In this case, differentiating (3.3) and combining with (2.6) yields

$$
y_{3}=\frac{\omega_{c}-\omega_{0}}{K_{v}}+\frac{1}{K_{v}} \dot{\alpha}
$$

Equation (3.4) can also be obtained by setting $\dot{\phi} \equiv 0$ in the first equation of system (3.1). Thus, during phase-lock, the LPF output gives the demodulation (up to additive and multiplicative constants) of an FM modulated input signal.

Let us assume that $y_{1}=f \circ \theta_{\text {in }}$ is an FM modulated signal, with instantaneous frequency

$$
\dot{\theta}_{\text {in }}(t)=\omega_{c}(1+M x(t)),
$$

where $\omega_{c}>0$ is the carrier frequency, $M \geq 0$ is a constant scaling of the modulation, and $x(t)$ is a bounded message signal of the form

$$
x(t)=h(\psi(t)),
$$

where $h: \mathbb{T}^{n} \rightarrow \mathbb{R}$ and $\psi: \mathbb{R} \rightarrow \mathbb{T}^{n}$ with $\dot{\psi}=\omega_{m} \in \mathbb{R}^{n}$ for some $n \in \mathbb{N}$. If the components of the vector $\omega_{m}$ are rationally related, then the message signal $x$ is a periodic function of $t$. Otherwise, $x$ is quasi-periodic. In either case, (3.2) becomes

$$
\ddot{\phi}+\frac{1}{\tau_{1}} \dot{\phi}+\frac{K_{0}}{\tau_{1}} \sin \phi=\omega_{c} M \dot{x}(t)+\frac{\omega_{c} M}{\tau_{1}} x(t)+\frac{\omega_{c}-\omega_{0}}{\tau_{1}} .
$$

If the input $y_{1}$ were an unmodulated sinusoid ( $M=0$, i.e., zero message), then the driving term would simply be a constant torque proportional to $\Delta \omega:=\omega_{c}-\omega_{0}$. In case $M=0$ and $\Delta \omega=0,(3.7)$ has the form of an undriven, damped pendulum. In this case, there are two fixed points: a stable fixed point at $(\phi, \dot{\phi})=(0,0)$ and a saddle point at $(\phi, \dot{\phi})=(\pi, 0)$. In case $0<|\Delta \omega|<K_{0}$, the two fixed points will be shifted towards each other, and for $|\Delta \omega|=K_{0}$ the two fixed points merge into one. For $|\Delta \omega|>K_{0}$, there are no fixed points; rather, this regime has a unique stable limit cycle (corresponding to the whirling pendulum). For small damping (approximately $\frac{1}{\tau_{1}}<\frac{\pi^{2}}{16} K_{0}$ ), this stable limit cycle persists even for $|\Delta \omega|<K_{0}$, until a homoclinic bifurcation occurs at $|\Delta \omega|=b$, where $b \approx \frac{4}{\pi} \sqrt{\frac{K_{0}}{\tau_{1}}}$ (see [9, p. 202], [22, Figure 8.5.10], or [12, Figure 3.8]). The region $b<|\Delta \omega|<K_{0}$ is bistable with two coexisting attractors: the stable fixed point and the stable limit cycle. For $|\Delta \omega|>K_{0}$ the limit cycle is

Copyright (c) by SIAM. Unauthorized reproduction of this article is prohibited. 
the only attractor, and for $|\Delta \omega|<b<K_{0}$ the stable fixed point is the only attractor. In the latter regime, phase-lock (i.e., $\dot{\phi} \equiv 0$ ) is approached asymptotically for all initial conditions. In the bistable regime, phase-lock is approached for those initial conditions in the basin of attraction of the fixed point, while phase-lock does not occur for initial conditions in the basin of attraction of the limit cycle. Using terminology from the PLL literature, we may call $2 K_{0}$ the width of the hold interval and $2 b \approx \frac{8}{\pi} \sqrt{\frac{K_{0}}{\tau_{1}}}$ the width of the pull-in interval. Note, however, that the analysis in this paragraph has been for the case $M=0$, i.e., no modulation or message in the input signal.

If modulation is added, for $M>0$ sufficiently small, the stable fixed point will be perturbed into a stable periodic orbit (if $x$ is periodic) or, more generally, an $n$-dimensional torus (if $x$ is quasi-periodic). In either case, phase-lock does not occur in the strict sense $(\dot{\phi} \equiv 0)$; rather, $\dot{\phi}$ will be near zero on the attracting limit cycle or torus. Thus, even in the simplified PLL model, for input signals with nonzero modulation (i.e., nonzero message), strict phase-lock will not be approached, even asymptotically. At best, a state of near phase-lock will be approached asymptotically.

In case the input phase $\theta_{\text {in }}$ satisfies (3.5) and (3.6), the simplified second-order ODE (3.7), or equivalently the system (3.1), may be expressed as the first-order autonomous system

$$
\begin{aligned}
\dot{\phi} & =-K_{v} y_{3}+\omega_{c} M h(\psi)+\omega_{c}-\omega_{0}, \\
K_{v} \dot{y}_{3} & =-\frac{K_{v}}{\tau_{1}} y_{3}+\frac{K_{0}}{\tau_{1}} \sin \phi, \\
\dot{\psi} & =\omega_{m} .
\end{aligned}
$$

4. Full model. Returning to the full model (2.15), or equivalently (2.16), we again consider an FM input signal with instantaneous frequency

$$
\dot{\theta}_{\text {in }}=\omega_{c}(1+M x(t)),
$$

where $\omega_{c}>0$ is the carrier frequency, $M \geq 0$ is a constant scaling of the modulation, and $x(t)$ is a quasi-periodic message signal of the form

$$
x(t)=h(\psi(t)),
$$

where $h: \mathbb{T}^{n} \rightarrow \mathbb{R}$ is bounded and $\psi: \mathbb{R} \rightarrow \mathbb{T}^{n}$ with $\dot{\psi}=\omega_{m} \in \mathbb{R}^{n}$ for some $n \in \mathbb{N}$.

The full system $(2.15)$ may be rewritten as the $(3+n)$-dimensional autonomous system

$$
\begin{aligned}
\dot{\phi} & =-K_{v} y_{3}+\omega_{c} M h(\psi)+\omega_{c}-\omega_{0}, \\
K_{v} \dot{y}_{3} & =-\frac{K_{v}}{\tau_{1}} y_{3}+\frac{K_{0}}{\tau_{1}}\left(\sin \phi+\sin \left(2 \theta_{\text {in }}-\phi\right)\right), \\
\dot{\psi} & =\omega_{m}, \\
\dot{\theta}_{\text {in }} & =\omega_{c}+\omega_{c} M h(\psi) .
\end{aligned}
$$

The full second-order ODE (2.16) for $\phi$ is

$$
\ddot{\phi}+\frac{1}{\tau_{1}} \dot{\phi}+\frac{K_{0}}{\tau_{1}}\left(\sin \phi+\sin \left(2 \theta_{\mathrm{in}}-\phi\right)\right)=\omega_{c} M D h\left(t \omega_{m}\right) \omega_{m}+\frac{\omega_{c} M}{\tau_{1}} h\left(\omega_{m} t\right)+\frac{\omega_{c}-\omega_{0}}{\tau_{1}} .
$$

Copyright (c) by SIAM. Unauthorized reproduction of this article is prohibited. 
In the strict phase-lock regime, the phase difference $\phi$ is constant (i.e., $\dot{\phi} \equiv 0$ ). In this case, in view of the first ODE in display (4.3) and the definition of $h$, the LPF output voltage is

$$
y_{3}=\frac{\omega_{c}}{K_{v}} M x(t)+\frac{\omega_{c}-\omega_{0}}{K_{v}} .
$$

Similar to the simplified model, if the PLL of the full model operates in strict phase-lock, then the LPF output is the demodulation of the FM modulated input signal (up to additive and multiplicative constants).

Unfortunately, strict phase-lock is unattainable in the full model, for all periodic or quasiperiodic messages of the form (4.2), including the case $M=0$ (in contrast to the simplified model). Indeed, by letting $Q=2 \theta_{\text {in }}-\phi$ in (4.4) and setting $\dot{\phi} \equiv 0$, we can see that strict phaselock could only be attained for the special class of messages $h$ for which $u(t):=\omega_{c} M h\left(\omega_{m} t\right)$ satisfies the ODE system

$$
\begin{aligned}
\dot{u} & =\frac{K_{0}}{\tau_{1}}(\sin \phi+\sin Q)-\frac{1}{\tau_{1}} u+\frac{\omega_{0}-\omega_{c}}{\omega_{c}}, \\
\dot{Q} & =2 \omega_{c}+2 u,
\end{aligned}
$$

in which $\phi$ is to be considered as a constant parameter. By the first equation of (4.6), every solution $u$ is bounded by a function of time that decays exponentially to zero, as can be seen by adding $u / \tau_{1}$ to both sides and making use of an integrating factor. However, $u(t):=\omega_{c} M h\left(\omega_{m} t\right)$ cannot approach zero if $h$ is periodic or quasi-periodic of the form (4.2), unless $M=0$ or $h \equiv 0$, in which case $u \equiv 0$. But $u \equiv 0$ cannot be a solution of (4.6), because in that case the first equation would imply that $Q$ is constant, while the second equation would imply that $Q$ is strictly increasing. Thus, the full PLL model does not allow strict phase-lock for all periodic or quasi-periodic messages of the form (4.2), even for the case of the zero message.

Although strict phase-lock is not possible, we will show that (under appropriate conditions) the PLL in the full model will tend to operate near the phase-locked regime (i.e., $\dot{\phi} \approx 0$ ), and (4.5) will be the dominant response of the LPF output, asymptotically in time.

5. Dimensionless model. The change of variables

$$
s=\omega_{0} t, \quad v=\frac{K_{v}}{\omega_{0}} y_{3}, \quad Q=2 \theta_{\text {in }}-\phi
$$

in system (4.3) yields the dimensionless model equations

$$
\begin{aligned}
\phi^{\prime} & =-v+\Omega_{c} M h(\psi)+\Omega_{c}-1, \\
v^{\prime} & =-a v+k \sin \phi+k \sin Q, \\
\psi^{\prime} & =\Omega, \\
Q^{\prime} & =v+\Omega_{c} M h(\psi)+\Omega_{c}+1,
\end{aligned}
$$

where

$$
a=\frac{1}{\omega_{0} \tau_{1}}, \quad k=\frac{K_{0}}{\omega_{0}^{2} \tau_{1}}, \quad \Omega_{c}=\frac{\omega_{c}}{\omega_{0}}, \quad \Omega=\frac{\omega_{m}}{\omega_{0}}
$$

Copyright (c) by SIAM. Unauthorized reproduction of this article is prohibited. 
and the prime denotes differentiation with respect to $s$.

The (positive) dimensionless quantities $a, k, M,\left|\Omega_{c}-1\right|$ and the components of the dimensionless vector $\Omega$ are typically much less than one. Taking advantage of this fact, we introduce the small parameter $\epsilon$ and write

$$
a=\alpha \epsilon^{2}, \quad k=\kappa \epsilon^{4}, \quad \Omega=\gamma \epsilon^{3}, \quad \Omega_{c}=1+\beta \epsilon^{3}, \quad M=m \epsilon^{3} .
$$

The powers of $\epsilon$ are chosen to facilitate the application of perturbation and averaging theory to the analysis of system (5.2). This choice is also consistent with the relative sizes of physically realistic parameter values. According to the LM565 PLL data sheet [17], its total loop gain is given by $K_{0}=33.6 f_{0} / V_{c}$, where $f_{0}$ is the VCO free running frequency in Hertz, $V_{c}$ is the total supply voltage, and the constant 33.6 is measured in Volts. As noted in the data sheet, for fixed $f_{0}$ and $V_{c}$, this total loop gain can be reduced by connecting a resistor between pins 6 and 7 of the package. With a total supply voltage of $V_{c}=24$ volts and an appropriate resistor between pins 6 and 7 to reduce the standard total loop gain by a factor of $1 / 2$, we have $K_{0}=0.7 f_{0}$. Choosing a typical value of $f_{0}=10 \mathrm{kHz}$, we obtain $K_{0}=7000 \mathrm{sec}^{-1}$ and $\omega_{0} \approx 62831.9 \mathrm{rad} / \mathrm{sec}$. Choosing an LPF time constant $\tau_{1}=1.8 \times 10^{-3} \mathrm{sec}$, and letting $\left|\omega_{m}\right|=100 \mathrm{rad} / \mathrm{sec}$ and $M=0.001$, then yields, approximately,

$$
a=0.008842, \quad k=0.000985, \quad|\Omega|=0.00159, \quad M=0.001,
$$

where $|\cdot|$ denotes a norm on $\mathbb{R}^{n}$. In this case, letting $\epsilon=0.1$ yields approximately

$$
\alpha=0.8842, \quad \kappa=9.85, \quad|\gamma|=1.59, \quad m=1,
$$

for the real parameters $\alpha, \kappa, m$, and the real $n$-dimensional vector $\gamma$.

Using the scaling (5.4), system (5.2) is recast in the form

$$
\begin{aligned}
\phi^{\prime} & =-v+\epsilon^{3}(m h(\psi)+\beta)+\epsilon^{6} \beta m h(\psi), \\
v^{\prime} & =-\epsilon^{2} \alpha v+\epsilon^{4} \kappa \sin \phi+\epsilon^{4} \kappa \sin Q \\
\psi^{\prime} & =\epsilon^{3} \gamma \\
Q^{\prime} & =2+v+\epsilon^{3}(m h(\psi)+\beta)+\epsilon^{6} \beta m h(\psi) .
\end{aligned}
$$

For $\epsilon>0$ and $z:=v / \epsilon^{2}$, system (5.5) becomes

$$
\begin{aligned}
\phi^{\prime} & =-\epsilon^{2} z+\epsilon^{3}(m h(\psi)+\beta)+\epsilon^{6} \beta m h(\psi), \\
z^{\prime} & =\epsilon^{2} \kappa \sin \phi+\epsilon^{2} \kappa \sin Q-\epsilon^{2} \alpha z, \\
\psi^{\prime} & =\epsilon^{3} \gamma, \\
Q^{\prime} & =2+\epsilon^{2} z+\epsilon^{3}(m h(\psi)+\beta)+\epsilon^{6} \beta m h(\psi),
\end{aligned}
$$

which is the correct form for averaging. Here, $\phi$ is the phase difference between the input signal and the VCO output, $z$ is a dimensionless rescaling of the LPF output, $\psi$ is the modulation phase, and $Q$ is the fast angle $2 \theta_{\text {in }}-\phi$ (i.e., the sum of the input and VCO output phases).

Copyright $\odot$ by SIAM. Unauthorized reproduction of this article is prohibited. 
Naively averaging system (5.6) over the fast angle $Q$ (that is, integrating the first three components of the vector field with respect to $Q$ over an interval of length $2 \pi$, which in this case amounts to dropping the term with factor $\sin Q$ ) yields the reduced system

$$
\begin{aligned}
\phi^{\prime} & =-\epsilon^{2} z+\epsilon^{3}(m h(\psi)+\beta)+\epsilon^{6} \beta m h(\psi), \\
z^{\prime} & =\epsilon^{2} \kappa \sin \phi-\epsilon^{2} \alpha z, \\
\psi^{\prime} & =\epsilon^{3} \gamma
\end{aligned}
$$

which is decoupled from $Q$ and equivalent to system (3.8) and the second-order ODE (3.7), obtained from physical arguments. A question naturally arises: to what extent do solutions of the naively averaged system (5.7) approximate solutions of the full system (5.6)?

A partial answer is provided by a more rigorous application of averaging theory. As illustrated in the next section, there exists a smooth near-identity transformation, of the form $I+O\left(\epsilon^{2}\right)$, that is $2 \pi$-periodic in $Q$ and that pushes the $Q$ dependence in the vector field of the first three equations in (5.6) to $O\left(\epsilon^{7}\right)$, without introducing new lower-order terms, so that at $O\left(\epsilon^{6}\right)$, the first three equations of the transformed system are decoupled from $Q$, and truncation to $O\left(\epsilon^{6}\right)$ yields the reduced system (5.7). Gronwall's inequality can be used to show that, on a time-scale of $1 / \epsilon^{2}$, solutions of the truncated system (5.7) approximate the first three components of solutions of the transformed full system to $O\left(\epsilon^{5}\right)$, which in turn are $O\left(\epsilon^{2}\right)$ close to the first three components of solutions of the original full system (5.6) (due to the near-identity transformation). Thus, solutions of (5.7) approximate the first three components of solutions of (5.6) to $O\left(\epsilon^{2}\right)$, on a time-scale of $1 / \epsilon^{2}$. This is a standard result (see [19]) of higher-order averaging (in this case, sixth-order averaging). However, it is important to note that in general, "naive" and rigorous higher-order averaging need not agree. The agreement in this case is a fortuitous consequence of the particular $\epsilon$-scaling chosen in (5.4), which allows for the existence of the near-identity transformation with all of the attributes described above.

We will extend the time-scale of the standard result stated above. Making use of attraction in the averaged system (5.7), we employ techniques from [20] to show that its solutions approximate the corresponding components of solutions of the full system (5.6) to $O\left(\epsilon^{2}\right)$ on an infinite forward time-scale. Furthermore, the averaging transformation itself yields high frequency correction terms that improve the approximation to $O\left(\epsilon^{5}\right)$, restoring some of the high frequency content that was lost in the averaging process, or equivalently, that was lost due to the physical assumption that the LPF perfectly attenuates signals with frequencies at the order of the carrier frequency.

6. Averaging transformation. Applying the (near identity) change of variables

$$
\begin{aligned}
\phi= & \bar{\phi}+\epsilon^{4} \frac{\kappa}{4} \sin Q-\epsilon^{6} \frac{\kappa}{8}(2 \bar{z} \sin Q-\alpha \cos Q), \\
z= & \bar{z}-\epsilon^{2} \frac{\kappa}{2} \cos Q+\epsilon^{4} \frac{\kappa}{4}(\alpha \sin Q+\bar{z} \cos Q)+\epsilon^{5} \frac{\kappa}{4}(m h(\psi)+\beta) \cos Q \\
& +\epsilon^{6} \frac{\kappa}{8}\left(\frac{\kappa}{2} \sin ^{2} Q+\left(\alpha^{2}-\bar{z}^{2}-\kappa \cos \bar{\phi}\right) \cos Q-(\alpha \bar{z}+\kappa \sin \bar{\phi}) \sin Q\right)
\end{aligned}
$$

Copyright $\odot$ by SIAM. Unauthorized reproduction of this article is prohibited. 
to (5.6) yields the equivalent system

$$
\begin{aligned}
\bar{\phi}^{\prime}= & -\epsilon^{2} \bar{z}+\epsilon^{3}(m h(\psi)+\beta)+\epsilon^{6} \beta m h(\psi)+O\left(\epsilon^{7}\right), \\
\bar{z}^{\prime}= & \epsilon^{2} \kappa \sin \bar{\phi}-\epsilon^{2} \alpha \bar{z}+O\left(\epsilon^{7}\right), \\
\psi^{\prime}= & \epsilon^{3} \gamma, \\
Q^{\prime}= & 2+\epsilon^{2} \bar{z}+\epsilon^{3}(m h(\psi)+\beta)-\epsilon^{4} \frac{\kappa}{2} \cos Q \\
& +\epsilon^{6} \beta m h(\psi)+\epsilon^{6} \frac{\kappa}{4}(\alpha \sin Q+\bar{z} \cos Q)+O\left(\epsilon^{7}\right),
\end{aligned}
$$

where dependence on the fast angle $Q$ in the vector field of the slow variables has been moved up from order two to seven in $\epsilon$, which allows decoupling from $Q$ after truncation at order six. The transformation (6.1) can be obtained in the usual manner by composing a series of transformations, each of which pushes the dependence on $Q$ in the action variables up by one order in epsilon (see [19]).

We let $(\rho, \zeta, \psi)$ denote the state variables for the truncation to order $\epsilon^{6}$ of the first three ODEs of the transformed system (6.2). The truncated system

$$
\begin{aligned}
\rho^{\prime} & =-\epsilon^{2} \zeta+\epsilon^{3}(m h(\psi)+\beta)+\epsilon^{6} \beta m h(\psi), \\
\zeta^{\prime} & =\epsilon^{2} \kappa \sin \rho-\epsilon^{2} \alpha \zeta, \\
\psi^{\prime} & =\epsilon^{3} \gamma
\end{aligned}
$$

is equivalent to system (5.7) obtained by naive averaging and system (3.8) obtained by physical reasoning.

Since the state spaces of the transformed system (6.2) and the truncated system (6.3) are, respectively, $\mathbb{T}^{1} \times \mathbb{R} \times \mathbb{T}^{n} \times \mathbb{T}^{1}$ and $\mathbb{T}^{1} \times \mathbb{R} \times \mathbb{T}^{n}$, where all but one of the state variables belong to compact manifolds, it can be easily verified that solutions of both systems exist for all forward time. Indeed, this result follows from the exponential decay of the single nonangular component $\bar{z}$ (respectively, $\zeta$ ) due to the damping term $-\epsilon^{2} \alpha \bar{z}$ (respectively, $-\epsilon^{2} \alpha \zeta$ ) in the second equation of system (6.2) (respectively, system (6.3)).

Every solution $(\rho, \zeta, \psi)$ of the truncated system (6.3) approximates the corresponding solution $(\bar{\phi}, \bar{z}, \psi, Q)$ of the transformed system (6.2) with the same initial data for its first three coordinates, by the estimate

$$
(\bar{\phi}, \bar{z}, \psi)=(\rho, \zeta, \psi)+O\left(\epsilon^{5}\right),
$$

on a time-scale of $1 / \epsilon^{2}$. This result can be verified by a standard application of Gronwall's inequality (see [19]). We will show that the time-scale can be extended from $1 / \epsilon^{2}$ to infinity.

To prove the time extension, we employ the idea introduced by Sanchez-Palencia in [18] and Sanders in [20]. They use contraction in the flow of the action variable of the truncated system to compensate for the exponential growth terms that arise in the standard Gronwall estimate of the difference between the solutions of the truncated and full systems. In [20], Sanders obtains the desired extension to infinite time for an action-angle system where the action variable of the truncated system has a hyperbolic attracting rest point, that is, where the truncated action-angle system has a hyperbolic attracting limit cycle. For sufficiently

Copyright (c) by SIAM. Unauthorized reproduction of this article is prohibited. 
small $\epsilon>0$, our truncated action system (6.3) has a hyperbolic attracting limit cycle (if the components of $\gamma$ are rationally related) or an $n$-dimensional torus (if $\gamma$ has components that are not rationally related). When it is coupled with the fast angle, our action-angle system has a hyperbolic attracting $(n+1)$-dimensional torus; thus, Sanders' result cannot be directly applied. Fortunately, the basic techniques used by Sanders for the limit cycle case can be modified to our flow with an attracting torus. The details of the proof are in Appendix A, Lemma A.1 and Theorem A.2, for an abstract system of greater generality than the PLL application discussed here. As a special case of these results, we have the following theorem.

Theorem 6.1. For sufficiently small $\epsilon>0$, there exists an $\epsilon$-independent product neighborhood $D_{0} \times \mathbb{T}^{n} \subset \mathbb{T}^{1} \times \mathbb{R} \times \mathbb{T}^{n}$ such that if $(\rho, \zeta, \psi)$ is a solution of the truncated system (6.3) with initial data in $D_{0} \times \mathbb{T}^{n}$ and $(\bar{\phi}, \bar{z}, \psi, Q)$ is a solution of the not truncated transformed system (6.2) with the same initial data (in the first three coordinates), then both solutions exist and satisfy the estimate

$$
(\bar{\phi}(s), \bar{z}(s), \psi(s))=(\rho(s), \zeta(s), \psi(s))+O\left(\epsilon^{5}\right)
$$

for all $s \geq 0$.

Using the transformation (6.1) to return to the original system yields higher-order correction terms for the approximation by the averaged system.

Corollary 6.2. For $\epsilon>0$ sufficiently small there exists a product neighborhood, $D_{0} \times \mathbb{T}^{n} \subset$ $\mathbb{T}^{1} \times \mathbb{R} \times \mathbb{T}^{n}$, such that if $(\rho, \zeta, \psi)$ is a solution of the truncated system (6.3) with initial data in $D_{0} \times \mathbb{T}^{n}$ and $(\phi, z, \psi, Q)$ is a solution of the original system (5.6) with the same initial data (in the first three coordinates), then both solutions exist for all forward time. Moreover, the solution of system (5.6) is approximated by the solution of (6.3) as follows:

$$
\begin{aligned}
\phi(s) & =\rho(s)+\epsilon^{4} \frac{\kappa}{4} \sin (Q(s))+O\left(\epsilon^{5}\right), \\
z(s) & =\zeta(s)-\epsilon^{2} \frac{\kappa}{2} \cos (Q(s))+\epsilon^{4} \frac{\kappa}{4}(\alpha \sin (Q(s))+\zeta(s) \cos (Q(s)))+O\left(\epsilon^{5}\right), \\
\psi(s) & =\epsilon^{3} \gamma s+\psi(0), \\
Q(s) & =2 \theta_{i n}(s)-\rho(s)+O\left(\epsilon^{4}\right),
\end{aligned}
$$

where

$$
\theta_{i n}(s)=\theta_{i n}(0)+\left(1+\beta \epsilon^{3}\right)\left(s+m \epsilon^{3} \int_{0}^{s} h(\psi(\xi)) d \xi\right) .
$$

We emphasize that by using a rigorous averaging transformation as opposed to naive averaging, we have not only shown that solutions of (5.6) are approximated by solutions of (6.3), but we have also obtained high frequency correction terms that improve the approximation.

The utility of the corollary in an application may depend on the extent to which the solutions of the truncated system (6.3) are known. In the next section, we show that for sufficiently small $\epsilon>0$ system (6.3) has an exponentially attracting invariant manifold consisting of periodic or quasi-periodic solutions that exhibit the expected behavior of the PLL: asymptotically, the dominant response of the LPF output is the demodulated message signal (up to a constant rescaling and shift).

Copyright (c) by SIAM. Unauthorized reproduction of this article is prohibited. 
7. Solution of the averaged system. In this section we use the persistence of normally hyperbolic invariant manifolds (see Appendix B) and perturbation theory to show that, for sufficiently small $\epsilon>0$, system (6.3) has an exponentially attracting invariant torus consisting of periodic or quasi-periodic solutions for which the dominant response of the LPF output, $\zeta$, is the demodulated message signal (up to a constant rescaling and shift).

Theorem 7.1. For sufficiently small $\epsilon>0$, the $O D E$ (6.3) has an n-dimensional exponentially attracting invariant torus $\mathcal{M}_{\epsilon}^{\text {avg }} \subset \mathbb{T}^{1} \times \mathbb{R} \times \mathbb{T}^{n}$ consisting of periodic or quasi-periodic orbits, and the corresponding solutions are approximated to $O\left(\epsilon^{3}\right)$ as stated in the formulas

$$
\begin{aligned}
\rho(s)= & \epsilon \frac{\alpha}{\kappa}(m h(\psi)+\beta)+\epsilon^{2} \frac{m}{\kappa}\left(1-\frac{\alpha^{2}}{\kappa}\right) D h(\psi) \gamma \\
& -\epsilon^{3}\left(\frac{\alpha m}{\kappa^{2}}\left(2-\frac{\alpha^{2}}{\kappa}\right) D^{2} h(\psi)(\gamma, \gamma)-\frac{1}{6}\left(\frac{\alpha}{\kappa}(m h(\psi)+\beta)\right)^{3}\right)+O\left(\epsilon^{4}\right), \\
\zeta(s)= & \epsilon(m h(\psi)+\beta)-\epsilon^{2} \frac{\alpha m}{\kappa} D h(\psi) \gamma-\epsilon^{3} \frac{m}{\kappa}\left(1-\frac{\alpha^{2}}{\kappa}\right) D^{2} h(\psi)(\gamma, \gamma)+O\left(\epsilon^{4}\right), \\
\psi(s)= & \epsilon^{3} \gamma s+\psi(0),
\end{aligned}
$$

where, for each $\psi \in \mathbb{T}^{n}, D^{2} h(\psi)(\cdot, \cdot): \mathbb{R}^{n} \times \mathbb{R}^{n} \rightarrow \mathbb{R}$ denotes the second derivative of $h$ at $\psi$ as a bilinear form.

Proof. Introducing the slow time $\tau=\epsilon^{2} s$, the truncated system (6.3) becomes

$$
\begin{aligned}
& \frac{d \rho}{d \tau}=-\zeta+\epsilon(m h(\psi)+\beta)+\epsilon^{4} \beta m h(\psi), \\
& \frac{d \zeta}{d \tau}=\kappa \sin \rho-\alpha \zeta \\
& \frac{d \psi}{d \tau}=\epsilon \gamma
\end{aligned}
$$

To make use of the theory of continuation of invariant manifolds as described in Appendix B, system (7.2) can be expressed in the more abstract form

$$
\begin{aligned}
x^{\prime} & =f(x)+H(x, \psi, \epsilon), \\
\psi^{\prime} & =Z(\psi, \epsilon),
\end{aligned}
$$

as in (B.1), where

$$
\begin{gathered}
x=\left(x_{1}, x_{2}\right)=(\rho, \zeta) \in \mathbb{T}^{1} \times \mathbb{R}, \quad \psi \in \mathbb{T}^{n}, \\
f(x)=\left(f_{1}\left(x_{1}, x_{2}\right), f_{2}\left(x_{1}, x_{2}\right)\right) \\
=\left(-x_{2}, \kappa \sin x_{1}-\alpha x_{2}\right), \\
H(x, \psi, \epsilon)=\left(H_{1}\left(x_{1}, x_{2}, \psi, \epsilon\right), H_{2}\left(x_{1}, x_{2}, \psi, \epsilon\right)\right) \\
=\left(\epsilon(m h(\psi)+\beta)+\epsilon^{4} \beta m h(\psi), 0\right),
\end{gathered}
$$

and

$$
Z(\psi, \epsilon)=\epsilon \gamma
$$

Copyright $\odot$ by SIAM. Unauthorized reproduction of this article is prohibited. 
In the unperturbed case, $\epsilon=0$, system (7.3) has a compact manifold of rest points, given by the trivial graph

$$
\mathcal{M}_{0}^{\text {avg }}=\left\{(0, \psi): \psi \in \mathbb{T}^{n}\right\} \subset \mathbb{T}^{1} \times \mathbb{R} \times \mathbb{T}^{n} .
$$

Note that the first derivative,

$$
D f(0):=\left[\begin{array}{ll}
\frac{\partial f_{1}}{\partial x_{1}}(0,0) & \frac{\partial f_{1}}{\partial x_{2}}(0,0) \\
\frac{\partial f_{2}}{\partial x_{1}}(0,0) & \frac{\partial f_{2}}{\partial x_{2}}(0,0)
\end{array}\right]=\left[\begin{array}{cc}
0 & -1 \\
\kappa & -\alpha
\end{array}\right],
$$

has eigenvalues $-\frac{\alpha}{2} \pm \frac{\sqrt{\alpha^{2}-4 \kappa}}{2}$, implying that $\mathcal{M}_{0}^{\text {avg }}$ is normally hyperbolic for $\alpha \neq 0$. That is, for $\epsilon=0$, the flow of (7.3) has exponential contraction $(\alpha>0)$ or expansion $(\alpha<0)$ in directions normal to $\mathcal{M}_{0}^{\text {avg }}$. (In the physical case of the PLL, $\alpha>0$ and thus $M_{0}$ is attracting.) By Proposition B.7 in Appendix B, the invariant manifold will persist under perturbation for sufficiently small $\epsilon>0$, and the perturbed manifold can be expressed as the graph

$$
\mathcal{M}_{\epsilon}^{\text {avg }}=\left\{\left(G^{\epsilon}(\psi), \psi\right): \psi \in \mathbb{T}^{n}\right\} \subset \mathbb{T}^{1} \times \mathbb{R} \times \mathbb{T}^{n},
$$

where the function $G^{\epsilon}: \mathbb{T}^{n} \rightarrow \mathbb{T}^{1} \times \mathbb{R}$ is smooth (to arbitrarily high order) both in its argument and in the parameter $\epsilon$. Thus, $G^{\epsilon}$ may be approximated by a series in $\epsilon$ to order $N$, with $N$ arbitrarily high:

$$
G^{\epsilon}(\psi)=G_{1}(\psi) \epsilon+G_{2}(\psi) \epsilon^{2}+G_{3}(\psi) \epsilon^{3}+\cdots+G_{N}(\psi) \epsilon^{N}+O\left(\epsilon^{N+1}\right),
$$

where $G_{j}: \mathbb{T}^{n} \rightarrow \mathbb{T}^{1} \times \mathbb{R}$ is a smooth function independent of $\epsilon$, for $j=1,2, \ldots, N$. By Proposition B.9, the perturbed manifold $\mathcal{M}_{\epsilon}^{\text {avg }}$ is also exponentially attracting. Since $\mathcal{M}_{\epsilon}^{\text {avg }}$ is a graph over $\psi$, every solution of (7.3) residing on $\mathcal{M}_{\epsilon}^{\text {avg }}$ is periodic (if the components of $\gamma$ are rationally related) or quasi-periodic (if $\gamma$ has components that are irrationally related).

The functions $\left\{G_{j}\right\}$ can be determined by making use of the invariance of the perturbed manifold $\mathcal{M}_{\epsilon}^{\text {avg }}$, where $x=G^{\epsilon}(\psi)$. Differentiation by the chain rule gives

$$
x^{\prime}=D G^{\epsilon}(\psi) \psi^{\prime},
$$

which, using (7.3), becomes

$$
f\left(G^{\epsilon}(\psi)\right)+H\left(G^{\epsilon}(\psi), \psi, \epsilon\right)=D G^{\epsilon}(\psi) Z(\psi, \epsilon) .
$$

To keep track of the components of this vector equation, we let

$$
G^{\epsilon}(\psi)=\left(\mu^{\epsilon}(\psi), \nu^{\epsilon}(\psi)\right)
$$

and

$$
G_{j}(\psi)=\left(\mu_{j}(\psi), \nu_{j}(\psi)\right), \quad j=1,2, \ldots, N .
$$

Combining (7.5)-(7.7) and (7.12)-(7.13) with (7.11), we obtain the system

$$
\begin{aligned}
-\nu^{\epsilon}(\psi)+\epsilon(m h(\psi)+\beta)+\epsilon^{4} \beta m h(\psi) & =\epsilon D \mu^{\epsilon}(\psi) \gamma, \\
\kappa \sin \left(\mu^{\epsilon}(\psi)\right)-\alpha \nu^{\epsilon}(\psi) & =\epsilon D \nu^{\epsilon}(\psi) \gamma .
\end{aligned}
$$

Copyright $\odot$ by SIAM. Unauthorized reproduction of this article is prohibited. 
Substitution of

$$
\begin{aligned}
& \mu^{\epsilon}(\psi)=\mu_{1}(\psi) \epsilon+\mu_{2}(\psi) \epsilon^{2}+\cdots+\mu_{N}(\psi) \epsilon^{N}+O\left(\epsilon^{N+1}\right), \\
& \nu^{\epsilon}(\psi)=\nu_{1}(\psi) \epsilon+\nu_{2}(\psi) \epsilon^{2}+\cdots+\nu_{N}(\psi) \epsilon^{N}+O\left(\epsilon^{N+1}\right)
\end{aligned}
$$

into (7.14), expansion, and collection of powers of $\epsilon$ yields a sequence of algebraic equations that can be solved for $\mu_{j}$ and $\nu_{j}, j=1, \ldots, N$. For $j=1,2,3$, we obtain

$$
\begin{aligned}
\mu_{1}(\psi) & =\frac{\alpha}{\kappa}(m h(\psi)+\beta), \\
\nu_{1}(\psi) & =m h(\psi)+\beta, \\
\mu_{2}(\psi) & =\frac{m}{\kappa}\left(1-\frac{\alpha^{2}}{\kappa}\right) D h(\psi) \gamma, \\
\nu_{2}(\psi) & =-\frac{\alpha m}{\kappa} D h(\psi) \gamma, \\
\mu_{3}(\psi) & =-\frac{\alpha m}{\kappa^{2}}\left(2-\frac{\alpha^{2}}{\kappa}\right) D^{2} h(\psi)(\gamma, \gamma)+\frac{1}{6}\left(\frac{\alpha}{\kappa}(m h(\psi)+\beta)\right)^{3}, \\
\nu_{3}(\psi) & =-\frac{m}{\kappa}\left(1-\frac{\alpha^{2}}{\kappa}\right) D^{2} h(\psi)(\gamma, \gamma) .
\end{aligned}
$$

Returning to previous notation, this yields the following approximation for every solution of (7.2) residing on the attracting manifold $\mathcal{M}_{\epsilon}^{\text {avg }}$ :

$$
\begin{aligned}
\rho(\tau)= & \epsilon \frac{\alpha}{\kappa}(m h(\psi)+\beta)+\epsilon^{2} \frac{m}{\kappa}\left(1-\frac{\alpha^{2}}{\kappa}\right) D h(\psi) \gamma \\
& -\epsilon^{3}\left(\frac{\alpha m}{\kappa^{2}}\left(2-\frac{\alpha^{2}}{\kappa}\right) D^{2} h(\psi)(\gamma, \gamma)-\frac{1}{6}\left(\frac{\alpha}{\kappa}(m h(\psi)+\beta)\right)^{3}\right)+O\left(\epsilon^{4}\right), \\
\zeta(\tau)= & \epsilon(m h(\psi)+\beta)-\epsilon^{2} \frac{\alpha m}{\kappa} D h(\psi) \gamma-\epsilon^{3} \frac{m}{\kappa}\left(1-\frac{\alpha^{2}}{\kappa}\right) D^{2} h(\psi)(\gamma, \gamma)+O\left(\epsilon^{4}\right), \\
\psi(\tau)= & \epsilon \gamma \tau+\psi(0)
\end{aligned}
$$

Changing back from the slow time $\tau$ to $s$ yields the conclusion of the theorem.

Recall that the LPF output in the averaged system is proportional to $\zeta$. Thus, on the attractor of the averaged system, the dominant response (first order in $\epsilon$ ) of the LPF output is proportional to $\epsilon(m h(\psi)+\beta)$, which is a rescaling of the message signal $h$, with an offset due to the detuning parameter $\beta$. The next-order correction term, $-\epsilon^{2} \frac{\alpha m}{\kappa} D h(\psi) \gamma$, has the effect of distorting the rescaled offset message. Increasing $\kappa$ (while holding all other parameters fixed) will decrease the magnitude of this second-order correction term as well as the third-order correction term. With $\epsilon$ fixed, an increase in $\kappa$ amounts to an increase in $k$, which can be achieved by increasing $K_{0}$, the total loop gain, while holding all other parameters fixed. This suggests that, in order to reduce distortion of the message appearing in the LPF output of the averaged system, the total loop gain, $K_{0}$, should be chosen as large as possible (or more specifically, large enough to make second- and third-order terms as small in magnitude as the fourth-order terms, assuming $\epsilon$ was chosen small enough so that each higher-order term was

Copyright (C) by SIAM. Unauthorized reproduction of this article is prohibited. 
originally smaller in magnitude than the previous one). In the next section, we show that the full system has an attractor on which the dominant response of the LPF output is the same, but that there are additional high frequency correction terms, leading to a suggested finite value of $K_{0}$ that will reduce distortion of the message.

8. Solution of the original system. Making the change of variables $\psi=\epsilon \eta$, the transformed full system (6.2) becomes

$$
\begin{aligned}
\bar{\phi}^{\prime}= & -\epsilon^{2} \bar{z}+\epsilon^{3}(m h(\epsilon \eta)+\beta)+\epsilon^{6} \beta m h(\epsilon \eta)+O\left(\epsilon^{7}\right), \\
\bar{z}^{\prime}= & \epsilon^{2} \kappa \sin \bar{\phi}-\epsilon^{2} \alpha \bar{z}+O\left(\epsilon^{7}\right), \\
\eta^{\prime}= & \epsilon^{2} \gamma \\
Q^{\prime}= & 2+\epsilon^{2} \bar{z}+\epsilon^{3}(m h(\epsilon \eta)+\beta)-\epsilon^{4} \frac{\kappa}{2} \cos Q \\
& +\epsilon^{6} \beta m h(\epsilon \eta)+\epsilon^{6} \frac{\kappa}{4}(\alpha \sin Q+\bar{z} \cos Q)+O\left(\epsilon^{7}\right) .
\end{aligned}
$$

As a special case of Theorem C.5 in Appendix C, we have the following proposition.

Proposition 8.1. For sufficiently small $\epsilon>0$, system (8.1) has an attracting invariant torus of dimension $n+1$, which is a continuation from the trivial manifold $\{0\} \times\{0\} \times \mathbb{T}^{n} \times \mathbb{T}^{1}$ at $\epsilon=0$.

Using the change of variables $\psi=\epsilon \eta$ to return to the full (transformed) system (6.2), we have the following corollary.

Corollary 8.2. System (6.2) has an attracting invariant torus of dimension $n+1$, which is arbitrarily close to the trivial manifold $\{0\} \times\{0\} \times \mathbb{T}^{n} \times \mathbb{T}^{1}$ for $\epsilon>0$ sufficiently small.

Returning to the original full system (5.6) by inverting the near-identity transformation (6.1), we obtain the following corollary.

Corollary 8.3. System (5.6) has an attracting invariant torus of dimension $n+1, \mathcal{M}_{\epsilon}^{\text {full }}$, which is arbitrarily close to the trivial manifold $\{0\} \times\{0\} \times \mathbb{T}^{n} \times \mathbb{T}^{1}$ for $\epsilon>0$ sufficiently small.

As a special case of Lemma A.1, we have the following contraction lemma for solutions of the averaged system (6.3).

Lemma 8.4. For $\epsilon>0$ sufficiently small, there exist constants $C_{1}>0, C_{2}>0$, and a product neighborhood, $D_{0} \times \mathbb{T}^{n} \subset \mathbb{T}^{1} \times \mathbb{R} \times \mathbb{T}^{n}$, such that for every $t_{0} \geq 0, t \geq t_{0}$, $\left(\rho_{0}, \zeta_{0}, \psi_{0}\right),\left(\hat{\rho}_{0}, \hat{\zeta}_{0}, \psi_{0}\right) \in D_{0} \times \mathbb{T}^{n}$, every pair of solutions $(\rho, \zeta, \psi)$ and $(\hat{\rho}, \hat{\zeta}, \psi)$ of the truncated system (6.3), with initial data $\left(\rho\left(t_{0}\right), \zeta\left(t_{0}\right), \psi\left(t_{0}\right)\right)=\left(\rho_{0}, \zeta_{0}, \psi_{0}\right)$ and $\left(\hat{\rho}\left(t_{0}\right), \hat{\zeta}\left(t_{0}\right), \psi\left(t_{0}\right)\right)=$ $\left(\hat{\rho}_{0}, \hat{\zeta}_{0}, \psi_{0}\right)$, satisfy the following contraction estimate:

$$
|(\rho(t), \zeta(t))-(\hat{\rho}(t), \hat{\zeta}(t))| \leq C_{1}\left|\left(\rho_{0}, \zeta_{0}\right)-\left(\hat{\rho}_{0}, \hat{\zeta}_{0}\right)\right| e^{-C_{2} \epsilon^{2}\left(t-t_{0}\right)}
$$

Combining these results with Corollary 6.2 and Theorem 7.1, we obtain an approximate asymptotic formula for solutions of the full system (5.6) on the attractor $\mathcal{M}_{\epsilon}^{\text {full }}$, as stated in the following corollary.

Corollary 8.5. For sufficiently small $\epsilon>0$, solutions of (5.6) on the attractor $\mathcal{M}_{\epsilon}^{\text {full }}$ are 
approximated to $O\left(\epsilon^{3}\right)$ by the following:

$$
\begin{aligned}
\phi(s)= & \frac{\alpha}{\kappa}(m h(\psi)+\beta)+\epsilon^{2} \frac{m}{\kappa}\left(1-\frac{\alpha^{2}}{\kappa}\right) D h(\psi) \gamma \\
& -\epsilon^{3}\left(\frac{\alpha m}{\kappa^{2}}\left(2-\frac{\alpha^{2}}{\kappa}\right) D^{2} h(\psi)(\gamma, \gamma)-\frac{1}{6}\left(\frac{\alpha}{\kappa}(m h(\psi)+\beta)\right)^{3}\right)+\delta_{1}(s)+O\left(\epsilon^{4}\right), \\
z(s)= & \epsilon(m h(\psi)+\beta)-\epsilon^{2}\left(\frac{\alpha m}{\kappa} D h(\psi) \gamma+\frac{\kappa}{2} \cos Q\right) \\
& -\epsilon^{3} \frac{m}{\kappa}\left(1-\frac{\alpha^{2}}{\kappa}\right) D^{2} h(\psi)(\gamma, \gamma)+\delta_{2}(s)+O\left(\epsilon^{4}\right), \\
\psi(s)= & \epsilon^{3} \gamma s+\psi(0), \\
Q(s)=2 \theta_{i n}(s)-\epsilon \frac{\alpha}{\kappa}(m h(\psi)+\beta)-\epsilon^{2} \frac{m}{\kappa}\left(1-\frac{\alpha^{2}}{\kappa}\right) D h(\psi) \gamma & +\epsilon^{3}\left(\frac{\alpha m}{\kappa^{2}}\left(2-\frac{\alpha^{2}}{\kappa}\right) D^{2} h(\psi)(\gamma, \gamma)-\frac{1}{6}\left(\frac{\alpha}{\kappa}(m h(\psi)+\beta)\right)^{3}\right)+O\left(\epsilon^{4}\right),
\end{aligned}
$$

where

$$
\theta_{\text {in }}(s)=\theta_{0}+\left(1+\beta \epsilon^{3}\right)\left(s+m \epsilon^{3} \int_{0}^{s} h(\psi(\xi)) d \xi\right)
$$

and $\left|\delta_{1}(s)\right|$ and $\left|\delta_{2}(s)\right|$ both approach zero exponentially as $s \rightarrow+\infty$.

Proof. For $\epsilon>0$ sufficiently small, both $\mathcal{M}_{\epsilon}^{\text {avg }} \times \mathbb{T}^{1}$ and $\mathcal{M}_{\epsilon}^{\text {full }}$ can be chosen to be arbitrarily close to $\{0\} \times\{0\} \times \mathbb{T}^{n} \times \mathbb{T}^{1}$, so that both lie well inside $D_{0} \times \mathbb{T}^{n} \times \mathbb{T}^{1}$, where the approximation given by Corollary 6.2 holds, and where the contraction lemma, Lemma 8.4 , can be applied.

Let $(\phi, z, \psi, Q)$ be a solution of the full system (5.6) with initial data on $\mathcal{M}_{\epsilon}^{\text {full }}$, and let $(\rho, \zeta, \psi)$ be the solution of the averaged system (6.3) with the same initial data (for the first three components). Let $(\hat{\rho}, \hat{\zeta}, \psi)$ denote a solution of the averaged system (6.3) with initial data on $\mathcal{M}_{\epsilon}^{\text {avg }}$, but with the same initial $\psi$ value. Note that all three solutions have the same $\psi$ component, namely $\psi(s)=\epsilon^{3} \gamma s+\psi(0)$. We then have

$$
(\phi, z)=(\hat{\rho}, \hat{\zeta})+((\phi, z)-(\rho, \zeta))+((\rho, \zeta)-(\hat{\rho}, \hat{\zeta}))
$$

The formulas given in (8.3) then follow from (8.5) by replacing $(\hat{\rho}, \hat{\zeta})$ with the formulas given by the first two equations of (7.1) in Theorem 7.1, replacing $(\phi, z)-(\rho, \zeta)$ by the correction terms given in the first two equations of (6.5) in Corollary 6.2, and bounding $|(\rho, \zeta)-(\hat{\rho}, \hat{\zeta})|$ by an exponentially decreasing function of time, as an application of the contraction lemma 8.4 (and using compactness of the attractors).

Recall that the LPF output is proportional to $z$. Thus, on the attractor in the full system, asymptotically in time, for $\epsilon$ sufficiently small, the dominant response (first-order in $\epsilon$ ) of the LPF output is the same as that of the averaged system: it is proportional to $\epsilon(m h(\psi)+\beta)$, a rescaling of the message signal with an offset due to the detuning parameter

Copyright (C) by SIAM. Unauthorized reproduction of this article is prohibited. 
$\beta$. However, the second-order correction term $-\epsilon^{2}\left(\frac{\alpha m}{\kappa} D h(\psi) \gamma+\frac{\kappa}{2} \cos Q\right)$ contains the high frequency contribution $-\epsilon^{2} \frac{\kappa}{2} \cos Q$, which would not appear in the solution of the simplified system obtained by heuristic physical reasoning or naive averaging. By using a rigorous averaging transformation, we have obtained an approximate solution that retains this high frequency contribution appearing in the original full system.

The high frequency contribution to the second-order correction term creates a significant distinction between the averaged and full systems in determining parameter values that will reduce distortion of the message in the LPF output. The absolute value of the second-order correction term in the full system is bounded by $\epsilon^{2}\left(\frac{c}{\kappa}+\frac{\kappa}{2}\right)$, where $c:=\alpha m\|D h\||\gamma|$, with $|\cdot|$ denoting the Euclidean norm and $\|\cdot\|$ denoting the sup norm. As a function of $\kappa$, this bound is minimized when $\kappa=\sqrt{2 c}$. In terms of physical parameters, this condition translates to a finite total loop gain value of $K_{0}=\omega_{0} \sqrt{2 M \tau_{1}\|D h\|\left|\omega_{m}\right|}$, which tends to minimize distortion of the message in the LPF output. Compare this with the averaged system, where the absolute value of the second-order correction term is bounded by $c / \kappa$, which is minimized by letting $\kappa$, and hence $K_{0}$, grow infinitely large.

With the appropriate change of variables to return from system (5.6) to the original system (4.3) in physical units, the dominant response of the LPF output, $z=\epsilon(m h(\psi)+\beta)$, transforms to

$$
y_{3}(t)=\frac{\omega_{0}}{K_{v}} M x(t)+\frac{\omega_{c}-\omega_{0}}{K_{v}},
$$

which is exactly the expression in (4.5) that is obtained heuristically by assuming that the PLL maintains phase-lock $(\dot{\phi} \equiv 0)$.

Appendix A. Time extension near an attracting torus. In [20], Sanders considered a system of ODEs in action-angle form expanded in a small parameter $\epsilon$ with one angle variable and $m$ action variables, where the coupling between the action variables and the angle variable occurs only in the highest-order term in $\epsilon$. With the presence of attraction in the lowest-order term of the action system, it was shown that an appropriate estimate of the difference between the action components of a solution of the truncated (and decoupled) system and the action components of the corresponding solution of the full system could be extended to all forward time under some hypotheses. In particular, the estimate was extended to all forward time when the truncated action-angle system contained a hyperbolic attracting limit cycle. We generalize this result by allowing for additional angle variables. Geometrically, this amounts to replacing the attracting limit cycle in the truncated system with a hyperbolic attracting multidimensional torus.

Let $l, m, n, K, N \in \mathbb{N}$ and assume $K \leq N$. Consider the full system

$$
\begin{aligned}
\dot{x} & =\epsilon^{K} F_{K}(x)+\sum_{p=K+1}^{N} \epsilon^{p} F_{p}(x, \psi, \epsilon)+\epsilon^{N+1} F_{N+1}(x, \psi, \theta, \epsilon), \\
\dot{\psi} & =G(\psi, \epsilon) \\
\dot{\theta} & =H(x, \psi, \theta, \epsilon),
\end{aligned}
$$

with the understanding that the sum from $K+1$ to $N$ does not appear in the first equation 
in case $K=N$, and the corresponding truncated system

$$
\begin{aligned}
\dot{x} & =\epsilon^{K} F_{K}(x)+\sum_{p=K+1}^{N} \epsilon^{p} F_{p}(x, \psi, \epsilon), \\
\dot{\psi} & =G(\psi, \epsilon), \\
\dot{\theta} & =\tilde{H}(x, \psi, \theta, \epsilon),
\end{aligned}
$$

where $x \in \mathbb{R}^{m}, \psi \in \mathbb{T}^{n}, \theta \in \mathbb{T}^{l}$, and $\epsilon \in[0,1]$. For the greatest generality in the following lemma and theorem, the truncation need be taken only in the $x$-component. The function $\tilde{H}$ could be the same as $H$, a truncation of $H$, or something completely different. However, the function $G$ must be the same in (A.1) and (A.2). In the application of our PLL model, the order of the $\epsilon$-dependence of $G$ does not exceed $N$. In this case, (A.2) could represent the truncation to order $N$ in $\epsilon$ of all components of the full system (A.1).

Lemma A.1 (contraction lemma). In system (A.2), suppose that the function $F_{K}$ is of class $C^{2}$ and that the functions $F_{p}, G$, and $\tilde{H}$ are of class $C^{1}$.

If $F_{K}(0)=0$ and all of the eigenvalues of the Jacobian matrix $D F_{K}(0)$ lie in the left-half of the complex plane, then there are positive constants $\epsilon_{0}<1, \alpha>0$, and $C>1$ (independent of $\epsilon$ ) and nested neighborhoods $D_{1} \subset D_{2} \subset D_{3}$ of the origin in $\mathbb{R}^{m}$ (independent of $\epsilon$ ) with $d_{1}:=\operatorname{dist}\left(D_{1}, \partial D_{2}\right)>0$ and $d_{2}:=\operatorname{dist}\left(D_{2}, \partial D_{3}\right)>0$ such that, for all $\epsilon \in\left[0, \epsilon_{0}\right]$, the manifold $D_{i} \times \mathbb{T}^{n} \times \mathbb{T}^{l}$ is forward invariant under (A.2) for each $i=1,2,3$.

Furthermore, for all $t_{0} \geq 0, t \geq t_{0}, x_{1}^{0}, x_{2}^{0} \in D_{3}, \psi^{0} \in \mathbb{T}^{n}$, and $\theta_{1}^{0}, \theta_{2}^{0} \in \mathbb{T}^{l}$, the two solutions $\left(x_{1}, \psi_{1}, \theta_{1}\right)$ and $\left(x_{2}, \psi_{2}, \theta_{2}\right)$ of (A.2) with initial conditions $\left(x_{1}\left(t_{0}\right), \psi_{1}\left(t_{0}\right), \theta_{1}\left(t_{0}\right)\right)=\left(x_{1}^{0}, \psi^{0}, \theta_{1}^{0}\right)$ and $\left(x_{2}\left(t_{0}\right), \psi_{2}\left(t_{0}\right), \theta_{2}\left(t_{0}\right)\right)=\left(x_{2}^{0}, \psi^{0}, \theta_{2}^{0}\right)$, respectively, satisfy the estimate

$$
\left|x_{1}(t)-x_{2}(t)\right| \leq C\left|x_{1}^{0}-x_{2}^{0}\right| e^{-\alpha \epsilon^{K}\left(t-t_{0}\right)} .
$$

In particular, there exist constants $0<k<1$ and $T>0$, independent of $\epsilon$, such that the contraction estimate

$$
\left|x_{1}\left(t+t_{0}\right)-x_{2}\left(t+t_{0}\right)\right| \leq k\left|x_{1}\left(t_{0}\right)-x_{2}\left(t_{0}\right)\right|
$$

holds whenever $t-t_{0} \geq T / \epsilon^{K}$.

Proof. The first equation of (A.2) may be rewritten as

$$
\dot{x}=\epsilon^{K} A x+\epsilon^{K} f(x)+\epsilon^{K+1} g(x, \psi, \epsilon),
$$

where $A:=D F_{K}(0), f(x):=F_{K}(x)-A x$, and

$$
g(x, \psi, \epsilon):=\sum_{p=K+1}^{N} \epsilon^{p-K-1} F_{p}(x, \psi, \epsilon) .
$$

As long as a solution $(x, \psi, \theta)$ of (A.2) exists, variation of parameters yields, for all $t_{0} \geq 0$ and $t \geq t_{0}$,

$$
x(t)=e^{\epsilon^{K}\left(t-t_{0}\right) A} x\left(t_{0}\right)+\int_{t_{0}}^{t} e^{\epsilon^{K}(t-s) A}\left(\epsilon^{K} f(x(s))+\epsilon^{K+1} g(x(s), \psi(s), \epsilon)\right) d s .
$$

Copyright (C) by SIAM. Unauthorized reproduction of this article is prohibited. 
Since the real parts of the eigenvalues of $A$ are all strictly negative, there exist positive constants $C$ and $\lambda$ such that, for all $s \in \mathbb{R}$,

$$
\left|e^{s A}\right| \leq C e^{-\lambda s}
$$

Since $D f(0)=0$, the continuity of the function $D f(x)=D F_{K}(x)-A$ with respect to $x$ at $x=0$ implies that there exists a constant $a>0$ sufficiently small (independent of $\epsilon$ ) such that

$$
L_{f}:=\sup _{x \in \mathbb{R}^{m},|x|<a}|D f(x)|<\frac{\lambda}{2 C} .
$$

By the compactness and continuity, we also have

$$
L_{g}:=\sup _{x \in \mathbb{R}^{m},|x|<a, \psi \in \mathbb{T}^{n}, \epsilon \in[0,1]}\left|D_{x} g(x, \psi, \epsilon)\right|<\infty,
$$

where $D_{x}$ denotes the derivative with respect to $x$ while holding $\psi$ fixed.

In view of the equations and inequalities (A.6)-(A.9), for $t \geq t_{0} \geq 0$, the first components of every pair of solutions $\left(x_{1}, \psi_{1}, \theta_{1}\right)$ and $\left(x_{2}, \psi_{2}, \theta_{2}\right)$ of system (A.2), with the same initial conditions for $\psi_{1}$ and $\psi_{2}$, satisfy the inequality

$$
\begin{aligned}
\left|x_{1}(t)-x_{2}(t)\right| \leq & \left|e^{\epsilon^{K}\left(t-t_{0}\right) A}\right|\left|x_{1}\left(t_{0}\right)-x_{2}\left(t_{0}\right)\right| \\
& +\left(\epsilon^{K} L_{f}+\epsilon^{K+1} L_{g}\right) \int_{t_{0}}^{t}\left|e^{\epsilon^{K}(t-s) A}\right|\left|x_{1}(s)-x_{2}(s)\right| d s \\
\leq & C e^{-\lambda \epsilon^{K}\left(t-t_{0}\right)}\left|x_{1}\left(t_{0}\right)-x_{2}\left(t_{0}\right)\right| \\
& +\left(\epsilon^{K} L_{f}+\epsilon^{K+1} L_{g}\right) C \int_{t_{0}}^{t} e^{-\lambda \epsilon^{K}(t-s)}\left|x_{1}(s)-x_{2}(s)\right| d s
\end{aligned}
$$

as long as both solutions exist; therefore, on the corresponding time interval,

$$
\begin{aligned}
e^{\lambda \epsilon^{K} t}\left|x_{1}(t)-x_{2}(t)\right| \leq & C e^{\lambda \epsilon^{K} t_{0}}\left|x_{1}\left(t_{0}\right)-x_{2}\left(t_{0}\right)\right| \\
& +\left(\epsilon^{K} L_{f}+\epsilon^{K+1} L_{g}\right) C \int_{t_{0}}^{t} e^{\lambda \epsilon^{K} s}\left|x_{1}(s)-x_{2}(s)\right| d s .
\end{aligned}
$$

Gronwall's inequality implies

$$
e^{\lambda \epsilon^{K} t}\left|x_{1}(t)-x_{2}(t)\right| \leq C e^{\lambda \epsilon^{K} t_{0}}\left|x_{1}\left(t_{0}\right)-x_{2}\left(t_{0}\right)\right| e^{\left(\epsilon^{K} L_{f}+\epsilon^{K+1} L_{g}\right) C\left(t-t_{0}\right)},
$$

and thus

$$
\begin{aligned}
\left|x_{1}(t)-x_{2}(t)\right| & \leq C\left|x_{1}\left(t_{0}\right)-x_{2}\left(t_{0}\right)\right| e^{-\left(\left(\lambda-L_{f} C\right)-L_{g} C \epsilon\right) \epsilon^{K}\left(t-t_{0}\right)} \\
& \leq C\left|x_{1}\left(t_{0}\right)-x_{2}\left(t_{0}\right)\right| e^{-\left(\lambda / 2-L_{g} C \epsilon\right) \epsilon^{K}\left(t-t_{0}\right)}
\end{aligned}
$$

where in the last inequality above we have used (A.8). If $\epsilon_{0}>0$ is sufficiently small so that

$$
\epsilon_{0} L_{g}<\frac{\lambda}{2 C}
$$

Copyright $\odot$ by SIAM. Unauthorized reproduction of this article is prohibited. 
then for $\epsilon \in\left[0, \epsilon_{0}\right]$ inequality (A.12) implies that

$$
\left|x_{1}(t)-x_{2}(t)\right| \leq C\left|x_{1}\left(t_{0}\right)-x_{2}\left(t_{0}\right)\right| e^{-\alpha \epsilon^{K}\left(t-t_{0}\right)},
$$

where

$$
\alpha:=\frac{\lambda}{2}-L_{g} C \epsilon_{0}>0
$$

For $\epsilon \in\left[0, \epsilon_{0}\right]$, the estimate (A.14) provides the desired contraction for as long as both solutions exist.

We will make use of a standard Lyapunov function (whose construction is given for completeness) to determine nested forward invariant sets for (A.2) where the inequality (A.14) is valid for all time.

Define the matrix $B:=\int_{0}^{\infty} e^{t A^{T}} e^{t A} d t$, where $A^{T}$ denotes the transpose of the matrix $A$. The integral converges since the matrices $A$ and $A^{T}$ each have the same eigenvalues, and thus each satisfies estimate (A.7). Note that $B^{T}=B$ and, for all $x \in \mathbb{R}^{m} \backslash\{0\}$,

$$
\begin{aligned}
\langle x, B x\rangle & =\int_{0}^{\infty}\left\langle x, e^{t A^{T}} e^{t A} x\right\rangle d t \\
& =\int_{0}^{\infty}\left\langle e^{t A} x, e^{t A} x\right\rangle d t \\
& >0
\end{aligned}
$$

where $\langle\cdot, \cdot\rangle$ is the standard Euclidean inner product in $\mathbb{R}^{m}$. Thus $B$ is a symmetric positivedefinite matrix. Define $V: \mathbb{R}^{m} \rightarrow \mathbb{R}$ by $V(x)=\langle x, B x\rangle$ for all $x \in \mathbb{R}^{m}$. Then $V$ induces the norm $|\cdot|_{V}$, defined by $|x|_{V}:=\sqrt{V(x)}$. The matrix $B$ satisfies Lyapunov's equation,

$$
A^{T} B+B A=-I,
$$

where $I$ is the $m \times m$ identity matrix. To verify this, note that

$$
\begin{aligned}
A^{T} B+B A & =\int_{0}^{\infty}\left(A^{T} e^{t A^{T}} e^{t A}+e^{t A^{T}} e^{t A} A\right) d t \\
& =\int_{0}^{\infty} \frac{d}{d t}\left(e^{t A^{T}} e^{t A}\right) d t \\
& =\lim _{t \rightarrow \infty} e^{t A^{T}} e^{t A}-I \\
& =-I,
\end{aligned}
$$

where in the last equation we have again used estimate (A.7) for both $A$ and $A^{T}$.

For all $x \in \mathbb{R}^{m}$,

$$
\begin{aligned}
f(x) & =F_{K}(x)-A x \\
& =\int_{0}^{1}(1-\tau)\left[D^{2} F_{K}(\tau x) \cdot x\right] \cdot x d \tau .
\end{aligned}
$$


Thus, for $|x|<a$, we have

$$
|f(x)| \leq \kappa|x|^{2}
$$

where $\kappa:=\sup _{x \in \mathbb{R}^{m},|x|<a}\left|D^{2} F_{K}(x)\right|<\infty$. Then for all $x \in \mathbb{R}^{m}$ with $|x| \leq a$ we have

$$
\begin{aligned}
\nabla V(x) \cdot F_{K}(x) & =\left\langle F_{K}(x), B x\right\rangle+\left\langle x, B F_{K}(x)\right\rangle \\
& =\left\langle x,\left(A^{T} B+B A\right) x\right\rangle+\langle f(x), B x\rangle+\langle x, B f(x)\rangle \\
& =-|x|^{2}+\langle f(x), B x\rangle+\langle x, B f(x)\rangle \\
& \leq|x|^{2}(-1+2 \kappa|B||x|) .
\end{aligned}
$$

In view of the inequality (A.21), for $r:=\min \left\{a, \frac{1}{4 \kappa|B|}\right\}$ and $x \in \mathbb{R}^{m}$ with $0<|x| \leq r$, we have

$$
\nabla V(x) \cdot F_{K}(x)<0
$$

Let $B_{r}(0):=\left\{x \in \mathbb{R}^{m}:|x|<r\right\}$ denote the open ball in $\mathbb{R}^{m}$ centered at the origin with radius $r$ in the usual Euclidean norm. Since the function $V$ is the square of the norm $|\cdot|_{V}$ (and all norms on $\mathbb{R}^{m}$ are equivalent), there exists $\rho>0$ sufficiently small such that

$$
\left\{x \in \mathbb{R}^{m}: V(x)<\rho\right\} \subset B_{r}(0) .
$$

Define

$$
D_{j}:=\left\{x \in \mathbb{R}^{m}: V(x)<\frac{\rho}{4-j}\right\}, \quad j=1,2,3 .
$$

Then $0 \in D_{1} \subset D_{2} \subset D_{3} \subset B_{r}(0)$, and $d_{j}:=\operatorname{dist}\left(D_{j}, \partial D_{j+1}\right)>0$ for $j=1,2$.

Note that, for $j=1,2,3, \partial D_{j}$ is a level set of $V$, and for all $x \in \partial D_{j}$ we have $\nabla V(x)$. $\epsilon^{K} F_{K}(x)<0$. By the continuity of the maps $x \mapsto \nabla V(x) \cdot F_{K}(x)$ and $(x, \psi, \epsilon) \mapsto \nabla V(x)$. $g(x, \psi, \epsilon)$, and the compactness of $\partial D_{j}, \mathbb{T}^{n}$, and $[0,1]$, there exists $\epsilon_{0}>0$ sufficiently small such that, for all $(x, \psi, \epsilon) \in \partial D_{j} \times \mathbb{T}^{n} \times\left[0, \epsilon_{0}\right]$, we have

$$
\nabla V(x) \cdot\left(F_{K}(x)+\epsilon g(x, \psi, \epsilon)\right)<0 .
$$

Thus, for $(x, \psi, \epsilon) \in \partial D_{j} \times \mathbb{T}^{n} \times\left(0, \epsilon_{0}\right]$,

$$
\nabla V(x) \cdot\left(\epsilon^{K} F_{K}(x)+\epsilon^{K+1} g(x, \psi, \epsilon)\right)<0 .
$$

Therefore, for $\epsilon \in\left[0, \epsilon_{0}\right], D_{j} \times \mathbb{T}^{n} \times \mathbb{T}^{l}$ is forward invariant under (A.2) for $j=1,2,3$.

If $t_{0} \geq 0, x_{1}^{0}, x_{2}^{0} \in D_{3}, \psi^{0} \in \mathbb{T}^{n}$, and $\theta_{1}^{0}, \theta_{2}^{0} \in \mathbb{T}^{l}$, then the two solutions $\left(x_{1}, \psi_{1}, \theta_{1}\right)$ and $\left(x_{2}, \psi_{2}, \theta_{2}\right)$ with initial conditions

$$
\left(x_{1}\left(t_{0}\right), \psi_{1}\left(t_{0}\right), \theta_{1}\left(t_{0}\right)\right)=\left(x_{1}^{0}, \psi^{0}, \theta_{1}^{0}\right)
$$

and

$$
\left(x_{2}\left(t_{0}\right), \psi_{2}\left(t_{0}\right), \theta_{2}\left(t_{0}\right)\right)=\left(x_{2}^{0}, \psi^{0}, \theta_{2}^{0}\right)
$$

respectively, satisfy the estimate (A.14) for all $t \geq t_{0}$. 
For $T>-\ln (1 / C) / \alpha$ and $k:=e^{-\alpha T}<1$, inequality (A.14) yields

$$
\left|x_{1}(t)-x_{2}(t)\right| \leq k\left|x_{1}\left(t_{0}\right)-x_{2}\left(t_{0}\right)\right|
$$

whenever $t-t_{0}>T / \epsilon^{K}$.

We remark that the contraction lemma, A.1, does not require that the eigenvalues of $D F_{K}(0)$ be distinct, as was assumed in [20].

Theorem A.2 (time extension). Suppose that in systems (A.1) and (A.2) the function $F_{K}$ is of class $C^{2}$ and the functions $F_{p}, G, H$, and $\tilde{H}$ are each of class $C^{1}$ for all integers $p$ such that $K+1 \leq p \leq N+1 ; F_{K}(0)=0$ and all eigenvalues of the Jacobian matrix $D F_{K}(0)$ lie in the left-half of the complex plane; and the domain $D_{1} \subset \mathbb{R}^{m}$ and the constants $\epsilon_{0}, k \in(0,1)$ are as in Lemma A.1.

Then, there exist constants $\epsilon_{1} \in\left(0, \epsilon_{0}\right]$ and $C_{1}>0$ such that for all $\epsilon \in\left[0, \epsilon_{1}\right]$, for all $x_{0}, \bar{x}_{0} \in D_{1}$ with $\left|x_{0}-\bar{x}_{0}\right|=O(\epsilon)$, and for all $\psi_{0} \in \mathbb{T}^{n}, \theta_{0}, \bar{\theta}_{0} \in \mathbb{T}^{l}$, the solution $(x, \psi, \theta)$ of (A.1) with initial condition $(x(0), \psi(0), \theta(0))=\left(x_{0}, \psi_{0}, \theta_{0}\right)$ and the solution $(\bar{x}, \bar{\psi}, \bar{\theta})$ of (A.2) with initial condition $(\bar{x}(0), \bar{\psi}(0), \bar{\theta}(0))=\left(\bar{x}_{0}, \psi_{0}, \bar{\theta}_{0}\right)$ satisfy the estimate

$$
|x(t)-\bar{x}(t)| \leq C_{1} \frac{1+k}{1-k}\left(\left|x_{0}-\bar{x}_{0}\right|+\epsilon^{N-K+1}\right)
$$

for all $t \geq 0$. In particular, if $x_{0}=\bar{x}_{0}$, then $|x(t)-\bar{x}(t)|=O\left(\epsilon^{N-K+1}\right)$ for all $t \geq 0$.

Proof. By the contraction lemma, A.1, there exist $\epsilon_{0} \in(0,1)$ and nested neighborhoods $0 \in D_{1} \subset D_{2} \subset D_{3} \subset \mathbb{R}^{m}$, each independent of $\epsilon$, with $d_{1}:=\operatorname{dist}\left(D_{1}, \partial D_{2}\right)>0$ and $d_{2}:=\operatorname{dist}\left(D_{2}, \partial D_{3}\right)>0$, such that for all $\epsilon \in\left[0, \epsilon_{0}\right], D_{i} \times \mathbb{T}^{n} \times \mathbb{T}^{l}$ is forward invariant under (A.2), for $i=1,2,3$. Furthermore, there exist constants $k \in(0,1)$ and $T>0$, independent of $\epsilon$, such that for all $\epsilon \in\left[0, \epsilon_{0}\right]$ and $t_{0} \geq 0$ every pair of solutions $\left(\bar{x}_{1}, \bar{\psi}_{1}, \bar{\theta}_{1}\right)$ and $\left(\bar{x}_{2}, \bar{\psi}_{2}, \bar{\theta}_{2}\right)$ of (A.2) with initial conditions $\bar{x}_{1}\left(t_{0}\right), \bar{x}_{2}\left(t_{0}\right) \in D_{3}, \psi_{1}\left(t_{0}\right)=\psi_{2}\left(t_{0}\right) \in \mathbb{T}^{n}$, and $\bar{\theta}_{1}\left(t_{0}\right), \bar{\theta}_{2}\left(t_{0}\right) \in \mathbb{T}^{l}$ satisfies the contraction estimate

$$
\left|\bar{x}_{1}\left(t+t_{0}\right)-\bar{x}_{2}\left(t+t_{0}\right)\right| \leq k\left|\bar{x}_{1}\left(t_{0}\right)-\bar{x}_{2}\left(t_{0}\right)\right|
$$

whenever $t-t_{0} \geq T / \epsilon^{K}$.

Let $\eta \in C^{\infty}\left(\mathbb{R}^{m},[0,1]\right)$ such that $\eta$ is unity on $D_{2}$ and zero on $\mathbb{R}^{m} \backslash D_{3}$. Consider the modified full system,

$$
\begin{aligned}
\dot{x} & =\eta(x)\left(\epsilon^{K} F_{K}(x)+\sum_{p=K+1}^{N} \epsilon^{p} F_{p}(x, \psi, \epsilon)+\epsilon^{N+1} F_{N+1}(x, \psi, \theta, \epsilon)\right), \\
\dot{\psi} & =G(\psi, \epsilon), \\
\dot{\theta} & =H(x, \psi, \theta, \epsilon),
\end{aligned}
$$

and the modified truncated system,

$$
\begin{aligned}
\dot{x} & =\eta(x)\left(\epsilon^{K} F_{K}(x)+\sum_{p=K+1}^{N} \epsilon^{p} F_{p}(x, \psi, \epsilon)\right), \\
\dot{\psi} & =G(\psi, \epsilon), \\
\dot{\theta} & =\tilde{H}(x, \psi, \theta, \epsilon) .
\end{aligned}
$$

Copyright (C) by SIAM. Unauthorized reproduction of this article is prohibited. 
Then $D_{3} \times \mathbb{T}^{n} \times \mathbb{T}^{l}$ is forward invariant under (A.28) and (A.29). Furthermore, on $D_{2} \times \mathbb{T}^{n} \times \mathbb{T}^{l}$, solutions of systems (A.1) and (A.28) are the same, and solutions of systems (A.2) and (A.29) are the same.

Let $\epsilon_{1} \in\left(0, \epsilon_{0}\right]$, to be further specified later. Let $\epsilon \in\left[0, \epsilon_{1}\right], x_{0}, \bar{x}_{0} \in D_{1}$ such that $\left|x_{0}-\bar{x}_{0}\right|=O(\epsilon), \psi_{0} \in \mathbb{T}^{n}$, and $\theta_{0}, \bar{\theta}_{0} \in \mathbb{T}^{l}$. Let $(x, \psi, \theta)$ be the solution of (A.28) with initial condition $(x(0), \psi(0), \theta(0))=\left(x_{0}, \psi_{0}, \theta_{0}\right)$, and let $(\bar{x}, \bar{\psi}, \bar{\theta})$ be the solution of (A.2) with initial condition $(\bar{x}(0), \bar{\psi}(0), \bar{\theta}(0))=\left(\bar{x}_{0}, \psi_{0}, \bar{\theta}_{0}\right)$. Since $D_{1} \times \mathbb{T}^{n} \times \mathbb{T}^{l}$ is forward invariant under (A.2) (by Lemma A.1), $(\bar{x}, \bar{\psi}, \bar{\theta})$ is also a solution of (A.29).

Integration of (A.28) and (A.29) yields, for all $t_{0} \geq 0$ and $t \geq t_{0}$,

$$
\begin{aligned}
x(t)= & x\left(t_{0}\right)+\int_{t_{0}}^{t} \eta(x(s)) \epsilon^{K} F_{K}(x(s)) d s \\
& +\int_{t_{0}}^{t} \eta(x(s)) \sum_{p=K+1}^{N} \epsilon^{p} F_{p}(x(s), \psi(s), \epsilon) d s \\
& +\int_{t_{0}}^{t} \eta(x(s)) \epsilon^{N+1} F_{N+1}(x(s), \psi(s), \theta(s), \epsilon) d s
\end{aligned}
$$

and

$$
\begin{aligned}
\bar{x}(t)= & \bar{x}\left(t_{0}\right)+\int_{t_{0}}^{t} \eta(\bar{x}(s)) \epsilon^{K} F_{K}(\bar{x}(s)) d s \\
& +\int_{t_{0}}^{t} \eta(\bar{x}(s)) \sum_{p=K+1}^{N} \epsilon^{p} F_{p}(\bar{x}(s), \bar{\psi}(s), \epsilon) d s .
\end{aligned}
$$

Since $\bar{\psi}(s)=\psi(s)$ for all $s \geq 0$, subtracting (A.31) from (A.30) and taking norms yields the following estimate, for all $t \geq t_{0}$ :

$$
\begin{aligned}
|x(t)-\bar{x}(t)| \leq & \left|x\left(t_{0}\right)-\bar{x}\left(t_{0}\right)\right|+\int_{t_{0}}^{t} \sum_{p=K}^{N} \epsilon^{p} L|x(s)-\bar{x}(s)| d s \\
& +\epsilon^{N+1}\left\|F_{N+1}\right\|\left(t-t_{0}\right),
\end{aligned}
$$

where $|\cdot|$ denotes the standard Euclidean norm, $\|\cdot\|$ denotes the sup norm over $D_{3} \times \mathbb{T}^{n} \times$ $\mathbb{T}^{l} \times[0,1]$, and

$$
L:=\max _{K \leq p \leq N, \epsilon \in[0,1]}\left\{\operatorname{Lip}\left(\eta F_{p}\right)\right\},
$$

where $\operatorname{Lip}\left(\eta F_{K}\right)$ denotes the Lipschitz constant of $\eta F_{K}$ over $D_{3}$, and for $K+1 \leq p \leq N$, $\operatorname{Lip}\left(\eta F_{p}\right)$ denotes the Lipschitz constant of $\eta F_{p}$ over $D_{3} \times \mathbb{T}^{n}$. Note that $L$ is independent of $\epsilon$.

Applying Gronwall's inequality to (A.32) yields, for all $t \geq t_{0}$,

$$
\begin{aligned}
|x(t)-\bar{x}(t)| & \leq\left(\left|x\left(t_{0}\right)-\bar{x}\left(t_{0}\right)\right|+\left\|F_{N+1}\right\|\left(t-t_{0}\right)\right) e^{L\left(t-t_{0}\right) \sum_{p=K}^{N} \epsilon^{p}} \\
& \leq\left(\left|x\left(t_{0}\right)-\bar{x}\left(t_{0}\right)\right|+\left\|F_{N+1}\right\|\left(t-t_{0}\right)\right) e^{L(N-K+1) \epsilon^{K}\left(t-t_{0}\right)} .
\end{aligned}
$$

Copyright (C) by SIAM. Unauthorized reproduction of this article is prohibited. 
Consider time intervals of the form $I_{j}:=\left[t_{j}, t_{j+1}\right]$, where $t_{j}:=j T / \epsilon^{K}$ for nonnegative integer $j$. Let $\left(\hat{x}_{j}, \hat{\psi}_{j}, \hat{\theta}_{j}\right)$ be the solution of the modified truncated system (A.29) with initial condition $\left(\hat{x}_{j}\left(t_{j}\right), \hat{\psi}_{j}\left(t_{j}\right), \hat{\theta}_{j}\left(t_{j}\right)\right)=\left(x\left(t_{j}\right), \psi\left(t_{j}\right), \theta\left(t_{j}\right)\right)$. Denoting the sup norm over $I_{j}$ by $\|\cdot\|_{I_{j}}$, we will use the triangle inequality and estimates of $\left\|x-\hat{x}_{j}\right\|_{I_{j}}$ and $\left\|\hat{x}_{j}-\bar{x}\right\|_{I_{j}}$ to obtain a recursive estimate of $\|x-\bar{x}\|_{I_{j}}$ in terms of $\|x-\bar{x}\|_{I_{j-1}}$.

Recall that $D_{3} \times \mathbb{T}^{n} \times \mathbb{T}^{l}$ is forward invariant under (A.29), and note that $\hat{\psi}_{j}=\psi$ on $I_{j}$ for each nonnegative integer $j$. The same arguments leading to (A.33) therefore yield, for all $t \in I_{j}$

$$
\begin{aligned}
\left|x(t)-\hat{x}_{j}(t)\right| & \leq\left(\left|x\left(t_{j}\right)-\hat{x}_{j}\left(t_{j}\right)\right|+\epsilon^{N+1}\left\|F_{N+1}\right\|\left(t-t_{j}\right)\right) e^{L(N-K+1) \epsilon^{K}\left(t-t_{j}\right)} \\
& \leq \epsilon^{N-K+1}\left\|F_{N+1}\right\| T e^{L(N-K+1) T} .
\end{aligned}
$$

Thus

$$
\left\|x-\hat{x}_{j}\right\|_{I_{j}} \leq C_{1} \epsilon^{N-K+1}
$$

where $C_{1}:=\max \left\{1, T\left\|F_{N+1}\right\|\right\} e^{L(N-K+1) T}$.

Since the map $t \mapsto\left|\bar{x}(t)-\hat{x}_{j}(t)\right|$ is continuous, there exists $t_{j}^{*} \in I_{j}$ such that

$$
\left\|\bar{x}-\hat{x}_{j}\right\|_{I_{j}}=\left|\bar{x}\left(t_{j}^{*}\right)-\hat{x}_{j}\left(t_{j}^{*}\right)\right| .
$$

Note that $t_{j}^{*}-T / \epsilon^{K} \in I_{j-1}$. By an application of the contraction estimate (A.27), we have

$$
\begin{aligned}
\left\|\bar{x}-\hat{x}_{j}\right\|_{I_{j}} & =\left|\bar{x}\left(t_{j}^{*}\right)-\hat{x}_{j}\left(t_{j}^{*}\right)\right| \\
& \leq k\left|\bar{x}\left(t_{j}^{*}-T / \epsilon^{K}\right)-\hat{x}_{j}\left(t_{j}^{*}-T / \epsilon^{K}\right)\right| \\
& \leq k\left\|\bar{x}-\hat{x}_{j}\right\|_{I_{j-1}}
\end{aligned}
$$

for all $j \in \mathbb{N}$.

Combining the triangle inequality, (A.35), and (A.37), we have, for all $j \in \mathbb{N}$,

$$
\begin{aligned}
\|x-\bar{x}\|_{I_{j}} & \leq\|x-\hat{x}\|_{I_{j}}+\left\|\hat{x}_{j}-\bar{x}\right\|_{I_{j}} \\
& \leq C_{1} \epsilon^{N-K+1}+k\left\|\hat{x}_{j}-\bar{x}\right\|_{I_{j-1}} \\
& \leq C_{1} \epsilon^{N-K+1}+k\left(\left\|\hat{x}_{j}-x\right\|_{I_{j-1}}+\|x-\bar{x}\|_{I_{j-1}}\right) \\
& \leq C_{1} \epsilon^{N-K+1}+k\left(C_{1} \epsilon^{N-K+1}+\|x-\bar{x}\|_{I_{j-1}}\right) \\
& =C_{1}(k+1) \epsilon^{N-K+1}+k\|x-\bar{x}\|_{I_{j-1}} .
\end{aligned}
$$

Iteration of the above recursive estimate is used to obtain, for all $j \in \mathbb{N}$, the inequality

$$
\|x-\bar{x}\|_{I_{j}} \leq k C_{1}\left|x_{0}-\bar{x}_{0}\right|+C_{1}(k+1) \epsilon^{N-K+1} \sum_{p=0}^{j} k^{p} .
$$

This can be proved by induction as follows. From (A.33), we obtain the following estimate:

$$
\begin{aligned}
\|x-\bar{x}\|_{I_{0}} & \leq\left(\left|x_{0}-\bar{x}_{0}\right|+\epsilon^{N-K+1} T\left\|F_{N+1}\right\|\right) e^{L(N-K+1) T} \\
& \leq C_{1}\left(\left|x_{0}-\bar{x}_{0}\right|+\epsilon^{N-K+1}\right) .
\end{aligned}
$$

Copyright (C) by SIAM. Unauthorized reproduction of this article is prohibited. 
To verify that (A.39) is true for $j=1$, note that (A.38) combined with the above estimate implies

$$
\begin{aligned}
\|x-\bar{x}\|_{I_{1}} & \leq C_{1}(k+1) \epsilon^{N-K+1}+k\|x-\bar{x}\|_{I_{0}} \\
& \leq C_{1}(k+1) \epsilon^{N-K+1}+k C_{1}\left(\left|x_{0}-\bar{x}_{0}\right|+\epsilon^{N-K+1}\right) \\
& =k C_{1}\left|x_{0}-\bar{x}_{0}\right|+C_{1}(2 k+1) \epsilon^{N-K+1} \\
& \leq k C_{1}\left|x_{0}-\bar{x}_{0}\right|+C_{1}(k+1)^{2} \epsilon^{N-K+1} \\
& =k C_{1}\left|x_{0}-\bar{x}_{0}\right|+C_{1}(k+1) \epsilon^{N-K+1} \sum_{p=0}^{1} k^{p}
\end{aligned}
$$

For the induction hypothesis, assume that (A.39) is true for $j=j^{*}$ for some $j^{*} \in \mathbb{N}$. To verify that this implies that (A.39) is true for $j=j^{*}+1$, we again use (A.38), combined with the induction hypothesis, to obtain

$$
\begin{aligned}
\|x-\bar{x}\|_{I_{j *+1}} \leq & C_{1}(k+1) \epsilon^{N-K+1}+k\|x-\bar{x}\|_{j_{j^{*}}} \\
\leq & C_{1}(k+1) \epsilon^{N-K+1} \\
& +k\left(k C_{1}\left|x_{0}-\bar{x}_{0}\right|+C_{1}(k+1) \epsilon^{N-K+1} \sum_{p=0}^{j^{*}} k^{p}\right) \\
= & k^{2} C_{1}\left|x_{0}-\bar{x}_{0}\right|+C_{1}(k+1) \epsilon^{N-K+1} \sum_{p=0}^{j^{*}+1} k^{p} \\
\leq & k C_{1}\left|x_{0}-\bar{x}_{0}\right|+C_{1}(k+1) \epsilon^{N-K+1} \sum_{p=0}^{j^{*}+1} k^{p} .
\end{aligned}
$$

Therefore, by induction, (A.39) is true for all $j \in \mathbb{N}$.

Taking the sup over all $j \in \mathbb{N}$ in (A.39), we obtain

$$
\begin{aligned}
\|x-\bar{x}\|_{[0, \infty)} & =\sup _{j \in \mathbb{N}}\left\{\|x-\bar{x}\|_{I_{j}}\right\} \\
& \leq k C_{1}\left|x_{0}-\bar{x}_{0}\right|+C_{1}(k+1) \epsilon^{N-K+1} \sum_{p=0}^{\infty} k^{p} \\
& =k C_{1}\left|x_{0}-\bar{x}_{0}\right|+C_{1} \frac{1+k}{1-k} \epsilon^{N-K+1} \\
& \leq C_{1} \frac{1+k}{1-k}\left(\left|x_{0}-\bar{x}_{0}\right|+\epsilon^{N-K+1}\right) .
\end{aligned}
$$

Recall that $(\bar{x}, \bar{\psi}, \bar{\theta})$ is a solution of both the truncated system (A.2) and the modified truncated system (A.29) and resides in $D_{1} \times \mathbb{T}^{n} \times \mathbb{T}^{l}$ for all $t \geq 0$. Our remaining task is to ensure that $(x, \psi, \theta)$, which was defined as a solution of the modified full system (A.28), is also a solution of the original full system (A.1). Since $\left|x_{0}-\bar{x}_{0}\right|=O(\epsilon)$, there exists a constant $C_{0}>0$ such that

$$
\left|x_{0}-\bar{x}_{0}\right| \leq C_{0} \epsilon
$$

Copyright (C) by SIAM. Unauthorized reproduction of this article is prohibited. 
Combining this with (A.40), we have

$$
\begin{aligned}
\|x-\bar{x}\|_{[0, \infty)} & \leq C_{1} \frac{1+k}{1-k}\left(C_{0} \epsilon+\epsilon^{N-K+1}\right) \\
& \leq \epsilon C_{1} \frac{1+k}{1-k}\left(C_{0}+1\right) .
\end{aligned}
$$

Let $\epsilon_{1} \in\left(0, \epsilon_{0}\right]$ be sufficiently small such that

$$
\epsilon_{1} C_{1} \frac{1+k}{1-k}\left(C_{0}+1\right) \leq \frac{d_{1}}{2}
$$

Then, for $\epsilon \in\left[0, \epsilon_{1}\right]$, we have $\|x-\bar{x}\|_{[0, \infty)} \leq d_{1} / 2$. Since $\bar{x}(t) \in D_{1}$ for all $t \geq 0$, inequality (A.42) implies that $x(t) \in D_{2}$ for all $t \geq 0$. So $(x, \psi, \theta) \in D_{2} \times \mathbb{T}^{n} \times \mathbb{T}^{l}$ for all forward time, which implies that it is also a solution of (A.1). The conclusion of the theorem then follows from estimate (A.40).

Appendix B. Continuation of invariant manifolds. In practice, systems of the form

$$
\begin{aligned}
\dot{x} & =f(x)+H(x, \psi, \epsilon), \\
\dot{\psi} & =Z(\psi, \epsilon)
\end{aligned}
$$

are encountered, where the differential equation $\dot{x}=f(x)$ in $\mathbb{R}^{m}$ has a compact invariant set $\mathcal{S}$ (perhaps a rest point, a periodic orbit, or a torus), the functions $H$ and $Z$ are $2 \pi$-periodic in each component of the $n$-vector of angles $\psi$, the quantity $\epsilon$ is a small (perhaps vector) parameter,

$$
H(x, \psi, 0) \equiv 0,
$$

and all the functions $f, H$, and $Z$ are class $C^{\infty}$. Each component of the state variable $\psi$ is an angular variable that may be restricted to the interval $[0,2 \pi]$ with its end points identified; that is, $\psi$ is the usual coordinate on the $n$-dimensional torus $\mathbb{T}^{n}$.

Define

$$
t \mapsto(X(t, x, \psi, \epsilon), \Psi(t, \psi, \epsilon))
$$

to be the solution of system (B.1) with initial condition

$$
X(0, x, \psi, \epsilon)=x, \quad \Psi(0, \psi, \epsilon)=\psi,
$$

where, in a slight abuse of notation, $x$ and $\psi$ now denote points in $\mathbb{R}^{m}$ and $\mathbb{T}^{n}$ rather than functions of time. In other words,

$$
\phi_{t}(x, \psi)=(X(t, x, \psi, \epsilon), \Psi(t, \psi, \epsilon))
$$

is the flow of the system of differential equations.

Differential equations of the form (B.1) are special because their solutions are periodic with respect to the angular variables. This is the content of the next proposition.

Proposition B.1. Solutions of system (B.1) are $2 \pi$-periodic in each angular variable. 
Proof. Using the flow of the system and with $E$ one of the usual unit basis vectors in $\mathbb{R}^{n}$, define a new function of time by

$$
(\tilde{X}(t, x, \psi, \epsilon), \tilde{\Psi}(t, \psi, \epsilon)):=(X(t, x, \psi+2 \pi E, \epsilon), \Psi(t, \psi+2 \pi E, \epsilon)) .
$$

This new function is a solution of the ODE with initial condition

$$
(\tilde{X}(0, x, \psi, \epsilon), \tilde{\Psi}(0, \psi, \epsilon))=(x, \psi+2 \pi E) .
$$

Since $\psi+2 \pi E=\psi$ on the torus, the flow and this new solution have the same initial condition; hence, they are equal.

The compact manifold $\mathcal{M}:=\left\{(x, \psi): x \in \mathcal{S}\right.$ and $\left.\psi \in \mathbb{T}^{n}\right\}$ is an invariant torus for system (B.1) with $\epsilon=0$. A natural and basic problem is to determine sufficient conditions for $\mathcal{M}$ to continue with respect to $\epsilon$; that is, there is some open set $J$ containing the origin of the parameter space such that for each $\epsilon \in J$ there is an invariant manifold $\mathcal{M}_{\epsilon}$ homeomorphic to $\mathcal{M}$, this family of manifolds depends continuously on $\epsilon$, and $\mathcal{M}_{0}=\mathcal{M}$. This definition may of course be strengthened to require the manifolds and the continuation to be smooth. Also, a definition is required to make precise the notion of a family of manifolds depending continuously (or smoothly) on a parameter. While it is possible to define the desired continuity in general, often the definition is apparent in each case. For the system (B.1), the definition of continuity leads naturally to a simple proof of the existence of a continuation that does not require the machinery for an abstract proof. In the applications, the determination and verification of hypotheses that imply the desired continuation are often best accomplished using the structure inherent in the application.

B.1. Continuation when the normal dynamics has a rest point. The simplest, but still important, case occurs when in system (B.1) the differential equation $\dot{x}=f(x)$ has a rest point, which without loss of generality may be taken at the origin; that is, $f(0)=0$. The corresponding manifold $\mathcal{M}:=\left\{(0, \psi): \psi \in \mathbb{T}^{n}\right\}$ may be considered to be the graph of the zero function over the torus $\mathbb{T}^{n}$. This fact suggests seeking a continuation of $\mathcal{M}$ as a family of manifolds each given as the graph of a function over the torus, that is, the graph of a function which is $2 \pi$-periodic in each of its variables.

Let $C_{2 \pi}^{r}\left(\mathbb{T}^{n}\right)$ denote the Banach space of $C^{r}$ functions that are $2 \pi$-periodic in each variable and $\mathbb{R}^{m}$-valued on $\mathbb{T}^{n}$ with respect to the usual $C^{r}$ norm. For each $F \in C_{2 \pi}^{r}\left(\mathbb{T}^{n}\right)$ there is a corresponding graph that gives the manifold $\left\{(F(\psi), \psi): \psi \in \mathbb{T}^{n}\right\}$.

B.1.1. Existence. The time- $\tau$ transform $\Gamma: C_{2 \pi}^{r}\left(\mathbb{T}^{n}\right) \times \mathbb{R} \rightarrow C_{2 \pi}^{r}\left(\mathbb{T}^{n}\right)$ is defined by

$$
\Gamma(F, \epsilon)(\theta)=X(\tau, F(\Psi(-\tau, \theta, \epsilon)), \Psi(-\tau, \theta, \epsilon), \epsilon) .
$$

The function $\Gamma$ is class $C^{r}$. This fact follows from the $\Omega$-lemma using the compactness of $\mathbb{T}^{n}$ and the smoothness of the flow $\phi_{t}$. In fact, by the existence theory for ODEs, the flow of a differential equation is as smooth (in the initial data and parameters) as the vector field that defines the differential equation.

The existence of solutions of system (B.1) for all forward time is a technical problem that may be overcome by noting that we are only interested in the behavior of the flow in some neighborhood of the unperturbed invariant manifold $\mathcal{M}$. If necessary, we can consider

Copyright (c) by SIAM. Unauthorized reproduction of this article is prohibited. 
a bounded open neighborhood of $\mathcal{M}$ and redefine the vector field, using bump functions, to agree with the original system on this neighborhood and be identically zero in the complement of some larger neighborhood. The flow of the modified vector field is complete. A continued invariant manifold that lies in the first mentioned open neighborhood is invariant for the original vector field. For simplicity, we will assume that the original vector field is complete.

Using the time- $\tau$ transform, the displacement function $\delta: C_{2 \pi}^{r}\left(\mathbb{T}^{n}\right) \times \mathbb{R} \rightarrow C_{2 \pi}^{r}\left(\mathbb{T}^{n}\right)$ is defined by

$$
\delta(F, \epsilon)=\Gamma(F, \epsilon)-F .
$$

It should be clear that fixed points of the time- $\tau$ transform correspond to zeros of the displacement.

Proposition B.2. The graph corresponding to a fixed point of the time- $\tau$ transform is invariant under the time- $\tau$ advance map of the flow.

Proof. Suppose that $F$ is a fixed point of $\Gamma$, and consider the graph in $\mathbb{R}^{m} \times \mathbb{T}^{n}$ over $\mathbb{T}^{n}$ given by $G:=\left\{(F(\psi), \psi): \psi \in \mathbb{T}^{n}\right\}$. Choose an arbitrary point $(F(\psi), \psi)$ on $G$. The position of the time- $\tau$ advance of this point under the flow is

$$
z:=(X(\tau, F(\psi), \psi, \epsilon), \Psi(\tau, \psi, \epsilon)) .
$$

There is a $\theta$ such that

$$
\psi=\Psi(-\tau, \theta, \epsilon)
$$

Hence,

$$
\begin{aligned}
z & =(X(\tau, F(\Psi(-\tau, \theta, \epsilon)), \Psi(-\tau, \theta, \epsilon), \epsilon), \Psi(\tau, \Psi(-\tau, \theta, \epsilon), \epsilon)) \\
& =(\Gamma(F)(\theta), \theta) .
\end{aligned}
$$

Because $F$ is a fixed point of the time- $\tau$ transform, $z=(F(\theta), \theta)$; therefore, $z \in G$.

Suppose that $\mathcal{F}$ is a function space, $U$ is an open set in $\mathcal{F}, J$ is an open set in a Banach space containing the origin, $\delta: \mathcal{F} \times J \rightarrow \mathcal{F}$, and $\delta(F, 0)=0$. We say that $F$ uniquely continues in $U$ with respect to the parameter $\epsilon$ if there is a function $\beta: J \rightarrow U, \beta(0)=F$, and $\beta$ is unique in the sense that if $G \in U, \epsilon \in J$, and $\delta(G, \epsilon)=0$, then $G=\beta(\epsilon)$.

The implicit function theorem, in this language, is the following basic result.

Theorem B.3. Suppose that $\mathcal{F}$ is a Banach space, $U$ is an open set in $\mathcal{F}, J$ is an open set in a Banach space containing the origin, $\delta: \mathcal{F} \times J \rightarrow \mathcal{F}$, and $\delta(F, 0)=0$. The function $F$ uniquely continues if $D \delta(F, 0): \mathcal{F} \rightarrow \mathcal{F}$ (the derivative of the function $H \mapsto \delta(H, 0)$ evaluated at $F$ ) is invertible as a bounded linear transformation of $\mathcal{F}$.

Clearly, continuation of a function in $C_{2 \pi}^{r}\left(\mathbb{T}^{n}\right)$ defines continuation of the corresponding manifold given as its graph. The next lemma is a well-known result.

Proposition B.4. Suppose that system (B.1) has an invariant torus given as the graph of a function $F$ over the torus $\mathbb{T}^{n}$. If $F$ continues uniquely as a fixed point of a corresponding time$\tau$ transform, then every element of the family of graphs in the continuation is an invariant torus for system (B.1).

Proof. Recall that $\varphi_{t}$ denotes the flow of the system (B.1). Let $\mathcal{M}_{\epsilon}$ denote the graph of the continuation of $F$ at $\epsilon$. Because the continuation is with respect to the graph transform, we have that $\varphi_{\tau}\left(\mathcal{M}_{\epsilon}\right)=\mathcal{M}_{\epsilon}$, where the dependence of the flow on $\epsilon$ is suppressed. By

Copyright (c) by SIAM. Unauthorized reproduction of this article is prohibited. 
Proposition B.1, the image of $\mathcal{M}_{\epsilon}$ under the flow remains a graph over the torus. By the continuity of the flow, there is some (sufficiently small) time $T>0$ such that for all $|t| \leq T$ the graph $\varphi_{t}\left(\mathcal{M}_{\epsilon}\right)$ remains in an open subset $\mathcal{U}$ in the state space $\mathbb{R}^{m} \times \mathbb{T}^{n}$ contained in the set of all points on graphs of functions $F$ in the set $U$ where fixed points of the graph transform are unique. For each such $t$, the semigroup property of the flow and the time- $\tau$ invariance of $\mathcal{M}_{\epsilon}$ imply that

$$
\varphi_{\tau}\left(\varphi_{t}\left(\mathcal{M}_{\epsilon}\right)\right)=\varphi_{t}\left(\varphi_{\tau}\left(\mathcal{M}_{\epsilon}\right)\right)=\varphi_{t}\left(\mathcal{M}_{\epsilon}\right) .
$$

The last equation states that $\varphi_{t}\left(\mathcal{M}_{\epsilon}\right)$ is time- $\tau$ invariant. By the uniqueness of the continuation, the manifold $\varphi_{t}\left(\mathcal{M}_{\epsilon}\right)$ must be the manifold $\mathcal{M}_{\epsilon}$. That is, $\mathcal{M}_{\epsilon}$ is invariant under the time- $t$ map for every $|t| \leq T$. An arbitrary $t$ may be expressed as $t=k T+\sigma$ for some integer $k$ and $|\sigma|<T$. Using the semigroup property of the flow, it follows immediately that $\mathcal{M}_{\epsilon}$ is invariant for the time- $t$ map.

As a corollary of Theorem B.3 and Propositions B.2 and B.4, we have the following abstract result.

Proposition B.5. If $r \geq 1, \delta(F, 0)=0$, and zero does not belong to the spectrum of the operator $D \delta(F, 0)$ on the Banach space $C_{2 \pi}^{r}\left(\mathbb{T}^{n}\right)$, then the invariant manifold given by the graph of a function $F$ continues uniquely in $C_{2 \pi}^{r}\left(\mathbb{T}^{n}\right)$.

Proof. The condition $r \geq 1$ is all that is required to apply the implicit function theorem.

Returning to system (B.1) and in view of the context defined here, the main issue is to determine conditions on the invariant manifold $\mathcal{M}:=\left\{(0, \psi): \psi \in \mathbb{T}^{n}\right\}$ that imply its unique continuation. The first result is simply the representation of the derivative $D \delta(0,0)$ with respect to a variational equation derived from system (B.1).

Proposition B.6. The derivative $D \delta(0,0)$ of the displacement function for system (B.1) (with $f(0)=0$ ) is the operator on the Banach space $C_{2 \pi}^{r}\left(\mathbb{T}^{n}\right)$ given by

$$
(D \delta(0,0) F)(\psi)=e^{\tau D f(0)} F(\Psi(-\tau, \psi, 0))-F(\psi) .
$$

Proof. To compute the action of $D \delta(0,0)$ on tangent vectors (which are simply functions in the Banach space $C_{2 \pi}^{r}\left(\mathbb{T}^{n}\right)$ ), choose a function $F \in C_{2 \pi}^{r}\left(\mathbb{T}^{n}\right)$ and consider the curve $\sigma \mapsto \sigma F$ of functions in $C_{2 \pi}^{r}\left(\mathbb{T}^{n}\right)$ passing through the origin. The directional derivative in the direction $F$ is given by

$$
D \delta(0,0) F=\left.\frac{d}{d \sigma} \delta(\sigma F, 0)\right|_{\sigma=0} .
$$

Note that

$$
\delta(\sigma F, 0)(\psi)=X(\tau, \sigma F(\Psi(-\tau, \psi, 0)), \Psi(-\tau, \psi, 0), 0)-\sigma F(\psi) .
$$

By differentiating with respect to $\sigma$ and then setting $\sigma=0$, the directional derivative is

$$
(D \delta(0,0) F)(\psi)=X_{x}(\tau, 0, \Psi(-\tau, \psi, 0), 0) F(\Psi(-\tau, \psi, 0))-F(\psi) .
$$

The partial derivative $X_{x}$ can be determined by solving the variational equation

$$
\dot{W}=D f(X(t, 0, \Psi(-\tau, \psi, 0), 0)) W
$$

Copyright (c) by SIAM. Unauthorized reproduction of this article is prohibited. 
obtained by differentiating both sides of the equation

$$
\frac{d}{d t} X(t, x, \Psi(-\tau, \psi, 0), 0)=f(X(t, x, \Psi(-\tau, \psi, 0), 0))
$$

with respect to initial data, that is, with respect to $x$ and defining $W=X_{x}(t, 0, \Psi(-\tau, \psi, 0), 0)$. In addition, the initial condition for the variational equation is $W(0)=I$, where $I$ is the $m \times m$ identity. Because $f(0)=0$, the function $t \mapsto X(t, 0, \Psi(-\tau, \psi, 0), 0)$ is identically zero, and the variational initial value problem reduces to

$$
\dot{W}=D f(0) W, \quad W(0)=I .
$$

Its solution is $W(t)=e^{t D f(0)}$. Thus,

$$
X_{x}(\tau, 0, \Psi(-\tau, \psi, 0), 0)=e^{\tau D f(0)} .
$$

All the ingredients are in place to study the continuation of the invariant torus $\mathcal{M}=$ $\left\{(0, \psi): \psi \in \mathbb{T}^{n}\right\}$. The simplest case occurs when $Z(\psi, 0) \equiv 0$; that is, the invariant manifold $\mathcal{M}$ consists entirely of rest points.

Proposition B.7. If in system (B.1), $Z(\psi, 0) \equiv 0$ and $f(0)=0$, then the invariant manifold $\mathcal{M}=\left\{(0, \psi): \psi \in \mathbb{T}^{n}\right\}$ for the system with parameter value $\epsilon=0$ consists entirely of rest points. If, in addition, zero is not an eigenvalue of $D f(0)$, then $\mathcal{M}$ uniquely continues in the system with respect to the parameter $\epsilon$.

Proof. The hypothesis $Z(\psi, 0) \equiv 0$ ensures that the invariant manifold at $\epsilon=0$ consists entirely of rest points; in particular, $\Psi(t, \psi, 0)=\psi$ for all $t$. To complete the proof, it suffices to show that there is some $\tau>0$ such that one is not an eigenvalue of $e^{\tau D f(0)}$ or, equivalently, zero is not an eigenvalue of $\tau D f(0)$. The only possibility for the condition not to be true is for $D f(0)$ to have an eigenvalue $\beta i$ on the imaginary axis such that $\tau \beta$ is an integer multiple of $2 \pi$. Since $D f(0)$ has a finite number of eigenvalues, simply choose $\tau$ sufficiently small such that all the eigenvalues on the imaginary axis lie in the interval $(-2 \pi i, 2 \pi i)$.

When $\mathcal{M}$ does not consist entirely of rest points, the most important case for applications is translation flow on this torus. In the context of system (B.1) this means $f(0)=0$ and $Z(\psi, 0)$ is a constant vector; that is, the flow on the invariant torus $\mathcal{M}$ for $\epsilon=0$ is translation flow. For this case, suppose that $\omega \in \mathbb{R}^{n}$ and

$$
Z(\psi, 0)=2 \pi \omega .
$$

The flow is called a rational translation if every component of $\omega$ is a rational multiple of the same real number; it is called an irrational translation otherwise. For translation flow, the invariant torus $\mathcal{M}$ is normally hyperbolic if $D f(0)$ is infinitesimally hyperbolic; that is, every eigenvalue of $D f(0)$ lies off the imaginary axis. When $D f(0)$ has eigenvalues on the imaginary axis, there are three cases:

(1) Irrational flow on the invariant torus.

(2) Normally resonant rational flow on the invariant torus; that is, $\omega=a R$ for some real number $a$ and $n$-vector of rational numbers $R$, and there is an eigenvalue $i 2 \pi \beta$ of $D f(0)$ on the imaginary axis, a vector $k \in \mathbb{Z}^{n}$, an integer $\ell$, and a resonance relation

$$
\beta=\ell a+k \cdot \omega=a(\ell+k \cdot R) .
$$

Copyright $\odot$ by SIAM. Unauthorized reproduction of this article is prohibited. 
(3) Normally nonresonant rational flow on the invariant torus; that is, rational flow but no resonance relation exists.

Proposition B.8. For system (B.1), if $f(0)=0$ and $D f(0)$ is infinitesimally hyperbolic or there is normally nonresonant rational flow on the invariant torus $\mathcal{M}=\left\{(0, \psi): \psi \in \mathbb{T}^{n}\right\}$, then $\mathcal{M}$ uniquely continues in the system with respect to the parameter $\epsilon$.

Proof. It suffices to show that for some $\tau>0$ the number 1 is not in the spectrum of the operator $D \Gamma(0,0)$ acting on $C_{2 \pi}^{r}\left(\mathbb{T}^{n}\right)$ by

$$
(D \Gamma(0,0) F)(\theta) \mapsto e^{\tau D f(0)} F(\theta-\tau \gamma),
$$

where $\gamma:=2 \pi \omega$ is the translation vector as in display (B.4).

The functions in $C_{2 \pi}^{r}\left(\mathbb{T}^{n}\right)$ may be represented by Fourier series,

$$
F(\theta)=\sum_{k \in \mathbb{Z}^{n}} a_{k} e^{i k \cdot \theta}
$$

where $a_{k}$ is a complex $n$-dimensional vector for each $k$ in the lattice of $n$-tuples of integers $\mathbb{Z}^{n}$; in keeping with standard notation, $k \cdot \theta$ is the usual inner product of the vector of integers $k$ and the vector of angles $\theta$. The number 1 is not in the spectrum if the equation $D \Gamma(0,0) F-F=$ $G$ is uniquely solvable for $F \in C_{2 \pi}^{r}\left(\mathbb{T}^{n}\right)$ whenever $G \in C_{2 \pi}^{r}\left(\mathbb{T}^{n}\right)$. In the space of Fourier coefficients, the equation is given by

$$
\left(e^{-i \tau k \cdot \gamma} e^{\tau D f(0)}-I\right) a_{k}=b_{k} .
$$

Thus, the desired result follows if the matrix $e^{-i \tau k \cdot \gamma} e^{\tau D f(0)}-I$ is invertible for every $k \in \mathbb{Z}^{n}$ and the collection of inverses is uniformly bounded.

By inspection, 1 is not an eigenvalue of the matrix $e^{-i \tau k \cdot \gamma} e^{\tau D f(0)}$ when $D f(0)$ is infinitesimally hyperbolic.

In case $D f(0)$ is not infinitesimally hyperbolic but there is normally nonresonant rational flow, all eigenvalues on the imaginary axis have the form $2 \pi \beta i$ for some real number $\beta$ and $\gamma=2 \pi a R$, where $a$ is a positive real number and $R$ is an $n$-vector of rational numbers. Let $\tau=1 / a$. Then the set $\left\{e^{-i 2 \pi a \tau k \cdot R}: k \in \mathbb{Z}^{n}\right\}$ is finite. Moreover, the eigenvalues that happen to lie on the unit circle for the finite set of matrices $e^{-i 2 \pi a \tau k \cdot R} e^{\tau D f(0)}$ all belong to the set

$$
\left\{e^{i 2 \pi \tau a(\beta / a-k \cdot R)}: k \in \mathbb{Z}^{n} \text {, and } 2 \pi \beta i \text { is an eigenvalue of } D f(0)\right\} \text {. }
$$

The nonresonance condition implies that 1 is not an element of this set.

Remark. In view of the proof of Proposition B.8, if the flow is an irrational translation, then the eigenvalues of $D \Gamma(0,0)$ are dense in the unit circle. Thus, in case $D f(0)$ is not infinitesimally hyperbolic, the spectrum of $D \Gamma(0,0)$ contains the entire unit circle and in particular the number 1 is in its spectrum. In this case, the invariant torus does not always persist. Indeed, in the Hamiltonian case diophantine conditions on the eigenvalues of the flow on the invariant torus are needed to prove persistence. In cases where the invariant torus does persist in the Hamiltonian case the results of this section suggest that the persistence might be destroyed by some non-Hamiltonian perturbation. In the normally resonant case, invariant tori may break up into island chains of elliptic (or nodal) points and saddles where the stable and unstable manifolds of adjacent saddles do not coincide; that is, while some periodic orbits might continue, the torus may not. Thus, it seems that the hypotheses of Proposition B.8 might be necessary for persistence.

Copyright (c) by SIAM. Unauthorized reproduction of this article is prohibited. 
B.1.2. Attraction. In cases where the invariant torus $\mathcal{M}=\left\{(0, \psi): \psi \in \mathbb{T}^{n}\right\}$ continues for small $|\epsilon|$ the remaining problem is to determine whether its structures also continue. The results presented in this section show that the manifold structure continues and that the continuation is as smooth as can be expected in the (vector) parameter $\epsilon$. By well-known theory, normal hyperbolicity continues. This result can be easily proved without using all of the machinery of the abstract theory of normally hyperbolic invariant manifolds in some of the cases we have considered. For non-normally hyperbolic invariant tori, there is no expectation that their normally resonant structure continues. In fact, for a generic situation, the flow on the continued torus would likely consist of a finite set of asymptotically stable and saddletype periodic orbits with perhaps a chaotic invariant set with saddle structure. Moreover, the normal dynamics would likely be hyperbolic. Of course, there is no simple theory to cover all cases. The $\epsilon=0$ dynamics and the nature of the perturbation must be taken into account.

Proposition B.9. Suppose that in system (B.1) all eigenvalues of $D f(0)$ lie in the left half of the complex plane and $\mathcal{M}_{\epsilon}$ is a continuation of the invariant torus $\mathcal{M}:=\left\{(0, \psi): \psi \in \mathbb{T}^{n}\right\}$. If $|\epsilon|$ is sufficiently small, then $\mathcal{M}_{\epsilon}$ is an attractor with exponentially fast attraction. Define

Proof. Choose an open set $\mathcal{U} \subset \mathbb{R}^{m}$ containing the origin, and note that $\mathcal{M} \subset \mathcal{U} \times \mathbb{T}^{n}$.

$$
R(x)=f(x)-D f(0) x,
$$

and consider system (B.1) in the equivalent form

$$
\begin{aligned}
\dot{x} & =D f(0) x+R(x)+H(x, \psi, \epsilon), \\
\dot{\psi} & =Z(\psi, \epsilon) .
\end{aligned}
$$

Choose a class- $C^{\infty}$ bump function $\rho: \mathbb{R}^{m} \rightarrow[0,1]$ that is unity on $\mathcal{U}$ and zero on the complement of some bounded open superset of $\mathcal{U}$. The flow of the new system,

$$
\begin{aligned}
& \dot{x}=D f(0) x+\rho(x)(R(x)+H(x, \psi, \epsilon)), \\
& \dot{\psi}=Z(\psi, \epsilon),
\end{aligned}
$$

is complete; it agrees with the flow of the original system on $\mathcal{U} \times \mathbb{T}^{n}$; and every solution starting in $\mathcal{U}$ is bounded for the modified system.

As usual, define the flow in its obvious components to be

$$
(X(t, x, \psi, \epsilon), \Psi(t, \psi, \epsilon)) .
$$

Of course, by definition, $X(0, x, \psi, \epsilon)=x$ and $\Psi(0, \psi, \epsilon)=\psi$.

The manifolds in the continuation are graphs over the torus $\mathbb{T}^{n}$ of the form

$$
\left\{(F(\theta, \epsilon), \theta): \theta \in \mathbb{T}^{n}\right\}
$$

The flow on such a graph is given by

$$
(F(\Psi(t, \theta, \epsilon), \epsilon), \Psi(t, \theta, \epsilon)),
$$

where $X(t, F(\theta), \theta, \epsilon)=F(\Psi(t, \theta, \epsilon), \epsilon)$. For a point $(\xi, \theta)$ not necessarily on the graph, the distance between the trajectory starting at this point and the graph is no larger than

$$
|X(t, \xi, \theta, \epsilon)-X(t, F(\theta, \epsilon), \theta, \epsilon)| .
$$

Copyright $\odot$ by SIAM. Unauthorized reproduction of this article is prohibited. 
The proof is completed by showing that this quantity decreases exponentially fast to zero. The desired estimate is obtained by a standard procedure using Gronwall's inequality.

Rearrange the first equation of the system to the form

$$
\dot{x}-D f(0) x=R(x)+H(x, \psi, \epsilon),
$$

and note that an equivalent formulation is

$$
\frac{d}{d t}\left(e^{-t D f(0)} x\right)=e^{-t D f(0)}(R(x)+H(x, \psi, \epsilon)) .
$$

By integration, we have the integral equation

$$
x(t)=e^{t D f(0)} x(0)+\int_{0}^{t} e^{(t-s) D f(0)}(R(x(s))+H(x(s), \psi(s), \epsilon)) d s .
$$

For simplification of notation, define

$$
\begin{aligned}
G(x, \psi, \epsilon) & :=R(x)+H(x, \psi, \epsilon), \\
X_{1}(t) & :=X(t, \xi, \theta, \epsilon), \\
X_{2}(t) & :=X(t, F(\theta, \epsilon), \theta, \epsilon), \\
\Psi(t) & :=\Psi(t, \theta, \epsilon),
\end{aligned}
$$

and note that

$$
\begin{aligned}
& X_{1}(t)=e^{t D f(0)} \xi+\int_{0}^{t} e^{(t-s) D f(0)} G\left(X_{1}(s), \Psi(s), \epsilon\right) d s, \\
& X_{2}(t)=e^{t D f(0)} F(\theta, \epsilon)+\int_{0}^{t} e^{(t-s) D f(0)} G\left(X_{2}(s), \Psi(s), \epsilon\right) d s .
\end{aligned}
$$

Because the eigenvalues of $D f(0)$ are in the left half of the complex plane, there are positive numbers $\lambda$ and $C$ such that

$$
\left|e^{t D f(0)} v\right| \leq C e^{-\lambda t}|v|
$$

for every $v \in \mathbb{R}^{m}$ and every $t \geq 0$. Subtract $X_{2}$ from $X_{1}$, take absolute values, and use the estimate to obtain the inequality

$$
\begin{aligned}
\left|X_{1}-X_{2}\right| \leq & C e^{-\lambda t}|\xi-F(\theta, \epsilon)| \\
& +C \int_{0}^{t} e^{-\lambda(t-s)}\left|G\left(X_{1}(s), \Psi(s), \epsilon\right)-G\left(X_{2}(s), \Psi(s), \epsilon\right)\right| d s .
\end{aligned}
$$

Claim. There is a number $M>0$ that may be made as small as desired by restricting the size of $|\xi|$ and $|\epsilon|$ such that

$$
\left|G\left(X_{1}(s), \Psi(s), \epsilon\right)-G\left(X_{2}(s), \Psi(s), \epsilon\right)\right| \leq M\left|X_{1}(s)-X_{2}(s)\right| .
$$


Assume the claim is true. Use it to estimate the integrand on the right-hand side of inequality (B.7), multiply both sides of the resulting inequality by $e^{t \lambda}$, and apply Gronwall's inequality to obtain the estimate

$$
|X(t, \xi, \theta, \epsilon)-X(t, F(\theta, \epsilon), \theta, \epsilon)| \leq C|\xi-F(\theta, \epsilon)| e^{(C M-\lambda) t} .
$$

By the claim, $M$ can be made small enough so that $C M-\lambda<0$. Thus, if the claim is true, the desired exponentially fast attraction to the invariant manifold is proved.

The claim follows from several observations. Using a triangle estimate, the quantity $\left|G\left(X_{1}(s), \Psi(s), \epsilon\right)-G\left(X_{2}(s), \Psi(s), \epsilon\right)\right|$ does not exceed

$$
\left|R\left(X_{1}(s)\right)-R\left(X_{2}(s)\right)\right|+\left|H\left(X_{1}(s), \Psi(s), \epsilon\right)-H\left(X_{2}(s), \Psi(s), \epsilon\right)\right| .
$$

The function $H(x, \psi, \epsilon)$ vanishes at $\epsilon=0$; therefore, by Taylor's theorem, there is a function $\mathcal{H}$ such that

$$
H(x, \psi, \epsilon)=\mathcal{H}(x, \psi, \epsilon) \epsilon .
$$

The desired estimate to verify the claim for the second term in display (B.10) is made by using three facts: $\mathcal{H}$ is Lipschitz, the second and third arguments of $H$ are the same in the second term in display (B.10), and $|\epsilon|$ is a factor after making the usual Lipschitz estimate. The function $R$ in the first term of the display requires a slightly more complicated treatment. Again, by an argument using Taylor's theorem, $R(x)=r(x) x^{2}$, where $r(x)$ is a bilinear form acting on two copies of $x$, the latter abbreviated by $x^{2}$. To estimate the difference, it is possible to justify the following inequalities:

$$
\begin{aligned}
\left|r\left(X_{1}\right) X_{1}^{2}-r\left(X_{2}\right) X_{2}^{2}\right| \leq & \left|r\left(X_{1}\right) X_{1}^{2}-r\left(X_{2}\right) X_{1}^{2}\right|+\left|r\left(X_{2}\right) X_{1}^{2}-r\left(X_{2}\right) X_{2}^{2}\right| \\
\leq & \left|r\left(X_{1}\right)-r\left(X_{2}\right)\right|\left|X_{1}\right|^{2}+\left|r\left(X_{2}\right)\right|\left|X_{1}-X_{2}\right|^{2} \\
\leq & \left(\operatorname{Lip}[R]\left|X_{1}\right|^{2}\right)\left|X_{1}-X_{2}\right| \\
& +\left(\max [R]\left|X_{1}-X_{2}\right|\right)\left|X_{1}-X_{2}\right| .
\end{aligned}
$$

The factors enclosed in parentheses in the last inequality can be chosen to be as small as desired: $\operatorname{Lip}[R]$ is bounded and $\left|X_{1}\right|$ is small by the choice of $\mathcal{U}$, and $\left|X_{1}-X_{2}\right|$ is bounded and $\max [R]$ is small by the choice of $\mathcal{U}$.

While the exponential estimate (B.9) holds in $\mathcal{U}$, in case a cut-off function is employed, solutions may not stay in $\mathcal{U}$ where the modified dynamics agrees with the dynamics of the original differential equation. But, using the exponential estimate, an orbit starting at $\xi$ does not move further than $C|\xi-F(\theta, \epsilon)|$ from the perturbed invariant torus. Since $C$ does not depend on the initial condition, we may choose an open set contained in $\mathcal{U}$ and containing the perturbed invariant torus so that solutions starting in this smaller set stay in $\mathcal{U}$.

Appendix C. Continuation of invariant manifolds for transformed (but not truncated) systems with one fast angle and multiple slow angles. As an abstraction for the full (transformed) model for the PLL (8.1), consider the system

$$
\begin{aligned}
& \dot{J}=\epsilon^{k} f(J)+O\left(\epsilon^{k+1}\right), \\
& \dot{\eta}=\epsilon^{k} \gamma+O\left(\epsilon^{k+1}\right), \\
& \dot{\theta}=\omega(J, \eta)+O(\epsilon),
\end{aligned}
$$


with state variables $(J, \eta, \theta) \in \mathbb{R}^{m} \times \mathbb{T}^{n} \times \mathbb{T}^{1}$, parameter $\epsilon \in[0,1)$, and where $\gamma \in \mathbb{R}^{n}$ is a constant vector, $k$ is a positive integer, and $\omega \in C^{1}\left(\mathbb{R}^{m} \times \mathbb{T}^{n},\left[\omega_{0}, \infty\right)\right)$, where $\omega_{0}$ is a strictly positive real number. Systems of this form arise naturally in the averaging process, after transforming with a near-identity transformation to push the fast angle dependence to higher orders, but before truncating. In the case of the PLL application, we have $f(0)=0$ and that $D f(0)$ is invertible, with eigenvalues all distinct and located in the left half of the complex plane, and $\omega$ is a positive constant.

Our goal is to find conditions for which the invariant torus $\{0\} \times \mathbb{T}^{n} \times \mathbb{T}^{1}$ for $(\mathrm{C} .1)$ at $\epsilon=0$ continues under the perturbation $\epsilon>0$. One answer is provided by the following theorem.

Theorem C.1. Suppose that system (C.1) is $C^{1}, f(0)=0$, and $\omega(J, \eta) \geq \omega_{0}>0$ for all $(J, \eta) \in \mathbb{R}^{m} \times \mathbb{T}^{n}$. Additionally, suppose that at least one of the following hypotheses is satisfied:

(i) All the eigenvalues of $D f(0)$ have nonzero real parts.

(ii) There exists a real number $\nu$ such that all of the components of $\nu \gamma$ are rational and no eigenvalues of $D f(0)$ have the form $i N q_{2} /\left(\nu q_{1}\right)$ for an integer $N$, where $q_{1}$ is the least common denominator of the absolute values of all the components of $\nu \gamma$, and $q_{2}$ is the greatest common divisor of all of the components of $q_{1} \nu \gamma$.

(iii) $n=1$ and $D f(0)$ is invertible.

Then, for sufficiently small $\epsilon>0$, the system (C.1) has an invariant torus of dimension $n+1$ that is a continuation from the invariant torus $\{0\} \times \mathbb{T}^{n} \times \mathbb{T}^{1}$ at $\epsilon=0$.

Proof. Following the proof of Theorem 7.11 in [3, p. 532], let

$$
(X(t, J, \eta, \epsilon), Y(t, J, \eta, \epsilon), \Theta(t, J, \eta, \epsilon))
$$

denote the solution of (C.1) with initial conditions

$$
X(0, J, \eta, \epsilon)=J, \quad Y(0, J, \eta, \epsilon)=\eta, \quad \Theta(0, J, \eta, \epsilon)=0 .
$$

Lemma C.2. For every compact set $K \subset \mathbb{R}^{m}$, there exists an open interval $L$ containing 0 and a map $T: K \times \mathbb{T}^{n} \times L \rightarrow \mathbb{R}$ such that $T(J, \eta, 0)=2 \pi / \omega(J, \eta)$ and $\Theta(T(J, \eta, \epsilon), J, \eta, \epsilon)=2 \pi$ for all $J \in K, \eta \in \mathbb{T}^{n}, \epsilon \in L$.

Proof. Note that

$$
\begin{aligned}
\Theta(t, J, \eta, 0) & =\int_{0}^{t} \omega(X(s, J, \eta, 0), Y(s, J, \eta, 0)) d s \\
& =\omega(J, \eta) t,
\end{aligned}
$$

and in particular, $\Theta(2 \pi / \omega(J, \eta), J, \eta, 0)=2 \pi$. Define

$$
\mathcal{F}(t, J, \eta, \epsilon):=\Theta(t, J, \eta, \epsilon)-2 \pi \text {. }
$$

Then $\mathcal{F}(2 \pi / \omega(J, \eta), J, \eta, 0)=0$, and $\mathcal{F}_{t}(2 \pi / \omega(J, \eta), J, \eta, 0)=\omega(J, \eta) \geq \omega_{0}>0$. The conclusion of the lemma follows from the implicit function theorem and the compactness of $K \times \mathbb{T}^{n}$.

Let $K \subset \mathbb{R}^{m}$ be a compact set containing the origin, and let the interval $L$ and map $T$ be as in the above lemma. Define the parameterized family of Poincaré maps $P: K \times \mathbb{T}^{n} \times L \rightarrow$ $\mathbb{R}^{m} \times \mathbb{T}^{n}$ by

$$
P(J, \eta, \epsilon):=(X(T(J, \eta, \epsilon), J, \eta, \epsilon), Y(T(J, \eta, \epsilon), J, \eta, \epsilon)) .
$$

Copyright (c) by SIAM. Unauthorized reproduction of this article is prohibited. 
Expanding (C.3) in $\epsilon$ about $\epsilon=0$ yields

$$
P(J, \eta, \epsilon)=(J, \eta)+\epsilon^{k} \frac{2 \pi}{\omega(J, \eta)}(f(J), \gamma)+O\left(\epsilon^{k+1}\right) .
$$

Consider the graph of a $C^{r}$ function $\beta: \mathbb{T}^{n} \rightarrow \mathbb{R}^{m}$, that is, the set

$$
\mathcal{M}:=\left\{(\beta(\eta), \eta): \eta \in \mathbb{T}^{n}\right\},
$$

and note that $\mathcal{M}$ is diffeomorphic to $\mathbb{T}^{n}$. If $\mathcal{M}$ is invariant under the Poincaré map, then the original system has an invariant torus (the cross product $\mathcal{M}$ and the circle corresponding to $\theta$ ) of dimension $n+1$.

Note that for some fixed $\epsilon$ the image of a point $(\beta(\eta), \eta)$ on $\mathcal{M}$ under the Poincaré map is

$$
P(\beta(\eta), \eta, \epsilon)=(\beta(\eta), \eta)+\epsilon^{k} \frac{2 \pi}{\omega(\beta(\eta), \eta)}(f(\beta(\eta)), \gamma)+O\left(\epsilon^{k+1}\right) .
$$

This image lies on $\mathcal{M}$ in case

$$
\beta\left(\eta+\epsilon^{k} \frac{2 \pi}{\omega(\beta(\eta), \eta)} \gamma+O\left(\epsilon^{k+1}\right)\right)=\beta(\eta)+\epsilon^{k} \frac{2 \pi}{\omega(\beta(\eta), \eta)} f(\beta(\eta))+O\left(\epsilon^{k+1}\right),
$$

which is equivalent to

$$
0=-\epsilon^{k} \frac{2 \pi}{\omega(\beta(\eta), \eta)} D \beta(\eta) \cdot \gamma+\epsilon^{k} \frac{2 \pi}{\omega(\beta(\eta), \eta)} f(\beta(\eta))+O\left(\epsilon^{k+1}\right),
$$

where $D \beta(\eta)$ is the derivative of $\beta$ with respect to $\eta$, which may be viewed as an $m \times n$ Jacobian matrix of partial derivatives. Dividing both sides by $\epsilon^{k} 2 \pi / \omega(\beta(\eta), \eta)$, we have

$$
0=-D \beta(\eta) \cdot \gamma+f(\beta(\eta))+O(\epsilon) .
$$

A solution $\beta$ of (C.8) corresponds to a manifold given as the graph of $\beta$ over $\mathbb{T}^{n}$, which is fixed under the Poincaré map (C.3).

For each positive integer $r$, define the function space $\mathcal{C}(r, \gamma)$ to be the set of all $\alpha \in$ $C^{r-1}\left(\mathbb{T}^{n}, \mathbb{R}^{m}\right)$ such that the map

$$
\eta \mapsto\left(d_{v} D^{\mu} \alpha\right)(\eta)
$$

exists and is continuous at each $\eta \in \mathbb{T}^{n}$, for each $n$-dimensional multi-index $\mu$ with $|\mu|=r-1$, and for each vector $v \in \gamma^{\perp}$, where $d_{v}$ denotes the directional derivative in the direction $v$, defined by

$$
\left(d_{v} \alpha\right)(\eta):=\left.\frac{d}{d s}\right|_{s=0} \alpha(\eta+s v)
$$

Note that $\mathcal{C}(r, \gamma)$ is a Banach space with the norm

$$
\|\alpha\|_{\mathcal{C}(r, \gamma)}:=\|\alpha\|_{C^{r-1}}+\sup _{(\mu, v) \in S}\left\|d_{v} D^{\mu} \alpha\right\|_{C^{0}}
$$


where $S:=\left\{(\mu, v) \in \mathbb{N}^{n} \times \mathbb{R}^{n}:|\mu|=r-1, v \in \gamma^{\perp},|v|=1\right\}$. To verify that $\mathcal{C}(r, \gamma)$ is complete, it suffices to check that $\mathcal{C}(1, \gamma)$ is complete, since if $\left\{\alpha_{j}\right\}$ is a Cauchy sequence in $\mathcal{C}(r, \gamma)$, then $\left\{D^{\mu} \alpha_{j}\right\}$ is a Cauchy sequence in $\mathcal{C}(1, \gamma)$. The completeness of $\mathcal{C}(1, \gamma)$ can be verified by the following argument. If $\left\{\alpha_{j}\right\}$ is a Cauchy sequence in $\mathcal{C}(1, \gamma)$, then for every vector $v \in \mathbb{R}^{n},\left\{\alpha_{j}\right\}$ and $\left\{d_{v} \alpha_{j}\right\}$ are Cauchy sequences in the Banach space $C^{0}\left(\mathbb{T}, \mathbb{R}^{m}\right)$, so there exist $\alpha, \sigma \in C^{0}\left(\mathbb{T}^{n}, \mathbb{R}^{m}\right)$ such that $\alpha_{j} \stackrel{C^{0}}{\rightarrow} \alpha$ and $d_{v} \alpha_{j} \stackrel{C^{0}}{\rightarrow} \sigma$. This implies that $d_{v} \alpha=\sigma$, and hence $\alpha \in \mathcal{C}(1, \gamma)$.

Consider the function $\Gamma: C^{r}\left(\mathbb{T}^{n}, \mathbb{R}^{m}\right) \times L \rightarrow \mathcal{C}(r, \gamma)$, which maps $(\beta, \epsilon) \in C^{r}\left(\mathbb{T}^{n}, \mathbb{R}^{m}\right) \times L$ to the right-hand side of (C.8); that is, define

$$
\Gamma(\beta, \epsilon)(\eta):=-D \beta(\eta) \cdot \gamma+f(\beta(\eta))+O(\epsilon) .
$$

Note that $\Gamma(0,0)(\eta) \equiv 0$ and $\left(\Gamma_{\beta}(0,0) \beta\right)(\eta)=-D \beta(\eta) \cdot \gamma+D f(0) \beta(\eta)$. We will apply the implicit function theorem to obtain a unique function $\beta$ such that $\Gamma(\beta, \epsilon)(\eta) \equiv 0$ for $\epsilon$ sufficiently small. Then this function $\beta$ (which depends on $\epsilon$ ) determines a graph $\mathcal{M}_{\epsilon}:=$ $\left\{(\beta(\eta)), \eta: \eta \in \mathbb{T}^{n}\right\}$, which is invariant under the Poincaré map and is a continuation of the trivial manifold $\{0\} \times \mathbb{T}^{n}$ at $\epsilon=0$.

In order to apply the implicit function theorem, we will show that the operator $\Gamma_{\beta}(0,0)$ : $C^{r}\left(\mathbb{T}^{n}, \mathbb{R}^{m}\right) \rightarrow \mathcal{C}(r, \gamma)$, defined by the mapping $\beta \mapsto-D \beta \cdot \gamma+D f(0) \beta$, has a bounded inverse. For this it is sufficient to show that, given $\alpha \in \mathcal{C}(r, \gamma)$, the following PDE can be uniquely solved for $\beta$,

$$
-D \beta \cdot \gamma+A \beta=\alpha
$$

where $A:=D f(0)$, and that there exists a constant $C>0$ such that this unique solution $\beta$ satisfies $\|\beta\|_{C^{r}} \leq C\|\alpha\|_{\mathcal{C}(r, \gamma)}$.

For every $\eta \in \mathbb{T}^{n}$ and $t \in \mathbb{R}$ there exists a point $\eta_{0} \in \mathbb{T}^{n}$ such that $\eta=\eta_{0}+t \gamma$. On the other hand, for $\eta_{0} \in \mathbb{T}^{n}$ fixed, the set $\left\{\eta=\eta_{0}+t \gamma: t \in \mathbb{R}\right\}$ is a curve in $\mathbb{T}^{n}$, tangent to $\gamma$ at $\eta_{0}$. On this curve, the PDE (C.12) becomes

$$
-D \beta\left(\eta_{0}+t \gamma\right) \cdot \gamma+A \beta\left(\eta_{0}+t \gamma\right)=\alpha\left(\eta_{0}+t \gamma\right),
$$

or equivalently

$$
-\frac{d}{d t} \beta\left(\eta_{0}+t \gamma\right)+A \beta\left(\eta_{0}+t \gamma\right)=\alpha\left(\eta_{0}+t \gamma\right)
$$

Multiplying both sides by $-e^{-t A}$ and collecting the total derivative, we obtain

$$
\frac{d}{d t}\left(e^{-t A} \beta\left(\eta_{0}+t \gamma\right)\right)=-e^{-t A} \alpha\left(\eta_{0}+t \gamma\right) .
$$

Integrating both sides from 0 to $t$, multiplying by $e^{t A}$, and rearranging yields

$$
\beta\left(\eta_{0}+t \gamma\right)=e^{t A} \beta\left(\eta_{0}\right)-e^{t A} \int_{0}^{t} e^{-s A} \alpha\left(\eta_{0}+s \gamma\right) d s .
$$

Copyright (c) by SIAM. Unauthorized reproduction of this article is prohibited. 
Substituting $\eta_{0}=\eta-t \gamma$, we have

$$
\beta(\eta)=e^{t A} \beta(\eta-t \gamma)-e^{t A} \int_{0}^{t} e^{-s A} \alpha(\eta+(s-t) \gamma) d s .
$$

Note that (C.17) is equivalent to the PDE (C.12) for all $t \in \mathbb{R}$. If $\alpha$ and $\beta$ are functions on $\mathbb{T}^{n}$, then we have the periodicity conditions

$$
\alpha\left(\eta+2 \pi k E_{j}\right)=\alpha(\eta), \quad \beta\left(\eta+2 \pi k E_{j}\right)=\beta(\eta)
$$

for every integer $k$ and for $j=1, \ldots, n$, where $\left\{E_{1}, \ldots, E_{n}\right\}$ is the standard unit basis for $\mathbb{R}^{n}$.

First, consider case (ii) of the theorem, where there exists a real number $\nu$ such that $\nu \gamma$ has all rational components. Let $q_{1}$ be the least common denominator of the absolute values of all the components of $\nu \gamma$, and let $q_{2}$ be the greatest common divisor of all the components of the vector $q_{1} \nu \gamma$. Then the vector $\left(\nu q_{1} / q_{2}\right) \gamma$ has all integer components, and we have

$$
t^{*} \gamma=\sum_{j=1}^{n} k_{j} 2 \pi E_{j}
$$

for some integers $k_{1}, \ldots, k_{n}$, where $t^{*}:=2 \pi \nu q_{1} / q_{2}$. Letting $t=t^{*}$ in (C.17) and using (C.18)(C.19), we have

$$
\beta(\eta)=e^{t^{*} A} \beta(\eta)-e^{t^{*} A} \int_{0}^{t^{*}} e^{-s A} \alpha(\eta+s \gamma) d s
$$

or equivalently

$$
\left(I-e^{t^{*} A}\right) \beta(\eta)=-e^{t^{*} A} \int_{0}^{t^{*}} e^{-s A} \alpha(\eta+s \gamma) d s
$$

In case $A$ has no eigenvalues of the form $i N q_{2} /\left(\nu q_{1}\right)$, for an integer $N$, then $I-e^{t^{*} A}$ is invertible, and we have

$$
\beta(\eta)=-\left(I-e^{t^{*} A}\right)^{-1} e^{t^{*} A} \int_{0}^{t^{*}} e^{-s A} \alpha(\eta+s \gamma) d s .
$$

Therefore, every solution of the PDE (C.12) must be given by formula (C.22). In particular, if a solution of PDE (C.12) exists, then it must be unique. On the other hand, it can be verified that (C.22) does indeed satisfy the PDE (C.12). Therefore (C.22) is the unique solution of PDE (C.12). Furthermore, it follows from (C.22) that $\beta \in C^{r}\left(\mathbb{T}^{n}, \mathbb{R}^{m}\right)$, and there exists a uniform constant $C>0$ such that $\|\beta\|_{C^{r}} \leq C\|\alpha\|_{\mathcal{C}(r, \gamma)}$ (see Proposition C.4). This implies that the operator $\Gamma_{\beta}(0,0)$ has a bounded inverse, and we obtain the theorem under hypothesis (ii).

Next, consider case (i), where $\gamma$ has arbitrary real components and no eigenvalues of $A$ lie on the imaginary axis. Then there exist two $A$-invariant subspaces of $\mathbb{R}^{m}, E^{s}$ and $E^{u}$, such that $\mathbb{R}^{m}=E^{s} \oplus E^{u}$. Furthermore, there exist positive constants $C^{s}, C^{u}, \lambda^{s}, \lambda^{u}$ such that

$$
\left|e^{t A} v^{s}\right| \leq C^{s} e^{-\lambda^{s} t}\left|v^{s}\right|, \quad t \geq 0,
$$

Copyright (C) by SIAM. Unauthorized reproduction of this article is prohibited. 
and

$$
\left|e^{t A} v^{u}\right| \leq C^{u} e^{\lambda^{u} t}\left|v^{u}\right|, \quad t \leq 0,
$$

for all $v^{s} \in E^{s}, v^{u} \in E^{u}$, and $t \in \mathbb{R}$, where $|\cdot|$ denotes the Euclidean norm on $\mathbb{R}^{m}$ (see [3], for example).

After making the change of variables $\tau=t-s$ in (C.17), we obtain

$$
\beta(\eta)=e^{t A} \beta(\eta-t \gamma)-\int_{0}^{t} e^{\tau A} \alpha(\eta-\tau \gamma) d \tau .
$$

The functions $\alpha$ and $\beta$ have unique decompositions $\alpha=\alpha^{s}+\alpha^{u}$ and $\beta=\beta^{s}+\beta^{u}$, where the ranges of $\alpha^{s}$ and $\beta^{s}$ lie in $E^{s}$ and the ranges of $\alpha^{u}$ and $\beta^{u}$ lie in $E^{u}$. By linearity and the $A$-invariance of $E^{s}$ and $E^{u}$, the component pairs $\alpha^{s}, \beta^{s}$ and $\alpha^{u}, \beta^{u}$ must satisfy (C.25) separately, i.e.,

$$
\beta^{s}(\eta)=e^{t A} \beta^{s}(\eta-t \gamma)-\int_{0}^{t} e^{\tau A} \alpha^{s}(\eta-\tau \gamma) d \tau
$$

and

$$
\beta^{u}(\eta)=e^{t A} \beta^{u}(\eta-t \gamma)-\int_{0}^{t} e^{\tau A} \alpha^{u}(\eta-\tau \gamma) d \tau
$$

Letting $t \rightarrow+\infty$ in (C.26) and $t \rightarrow-\infty$ in (C.27), and making use of (C.23) and (C.24), we obtain

$$
\beta^{s}(\eta)=-\int_{0}^{\infty} e^{\tau A} \alpha^{s}(\eta-\tau \gamma) d \tau
$$

and

$$
\beta^{u}(\eta)=\int_{-\infty}^{0} e^{\tau A} \alpha^{u}(\eta-\tau \gamma) d \tau
$$

Therefore

$$
\begin{aligned}
\beta(\eta) & =\beta^{s}(\eta)+\beta^{u}(\eta) \\
& =\int_{-\infty}^{0} e^{\tau A} \alpha^{u}(\eta-\tau \gamma) d \tau-\int_{0}^{\infty} e^{\tau A} \alpha^{s}(\eta-\tau \gamma) d \tau
\end{aligned}
$$

We have shown that every solution of the PDE (C.12) must be given by formula (C.30). In particular, if a solution of PDE (C.12) exists, then it must be unique. On the other hand, it can be verified that (C.30) does indeed satisfy the PDE (C.12). Therefore (C.30) is the unique solution of PDE (C.12). Furthermore, it follows from (C.30) that $\beta \in C^{r}\left(\mathbb{T}^{n}, \mathbb{R}^{m}\right)$, and there exists a uniform constant $C>0$ such that $\|\beta\|_{C^{r}} \leq C\|\alpha\|_{\mathcal{C}(r, \gamma)}$ (see Proposition C.3). This implies that the operator $\Gamma_{\beta}(0,0)$ has a bounded inverse, and we obtain the theorem under hypothesis (i). 
Note that if $\nu \gamma$ has all rational components and if all the eigenvalues of $A$ have negative real parts, then the formulas for $\beta$ given by (C.22) and (C.30) are equal.

Finally, consider the case of hypothesis (iii), where $n=1, A$ is an invertible $m \times m$ matrix, and $\gamma$ is a real number. In this case, the periodicity condition (C.18) reduces to

$$
\alpha(\eta+2 \pi k)=\alpha(\eta), \quad \beta(\eta+2 \pi k)=\beta(\eta)
$$

for every integer $k$. Evaluating (C.17) at $t=2 \pi / \gamma$ and using (C.31), we obtain

$$
\beta(\eta)=e^{\frac{2 \pi}{\gamma} A} \beta(\eta)-e^{\frac{2 \pi}{\gamma} A} \int_{0}^{\frac{2 \pi}{\gamma}} e^{-s A} \alpha(\eta+s \gamma) d s
$$

or equivalently

$$
\left(I-e^{\frac{2 \pi}{\gamma} A}\right) \beta(\eta)=-e^{\frac{2 \pi}{\gamma} A} \int_{0}^{\frac{2 \pi}{\gamma}} e^{-s A} \alpha(\eta+s \gamma) d s .
$$

Since $A$ is invertible, zero is not an eigenvalue of $A$. Thus $\left(I-e^{\frac{2 \pi}{\gamma} A}\right)$ is invertible, and we have

$$
\beta(\eta)=-\left(I-e^{\frac{2 \pi}{\gamma} A}\right)^{-1} e^{\frac{2 \pi}{\gamma} A} \int_{0}^{\frac{2 \pi}{\gamma}} e^{-s A} \alpha(\eta+s \gamma) d s .
$$

We have shown that, under hypothesis (iii), every solution of the PDE (C.12) must be given by formula (C.34). In particular, if a solution of PDE (C.12) exists, then it must be unique. On the other hand, it can be verified that (C.34) does indeed satisfy the PDE (C.12). Therefore (C.34) is the unique solution of PDE (C.12). Furthermore, it follows from (C.34) that $\beta \in$ $C^{r}\left(\mathbb{T}^{1}, \mathbb{R}^{m}\right)$, and there exists a uniform constant $C>0$ such that $\|\beta\|_{C^{r}} \leq C \|_{\alpha \|_{\mathcal{C}(r, \gamma)} \text {. (Note }}$ that for $n=1, \mathcal{C}(r, \gamma)$ is equivalent to $C^{r-1}\left(\mathbb{T}^{1}, \mathbb{R}^{m}\right)$.) This implies that the operator $\Gamma_{\beta}(0,0)$ has a bounded inverse, and we obtain the theorem under hypothesis (iii).

Proposition C.3. Suppose that the vector $\gamma \in \mathbb{R}^{n}$, let $\alpha \in \mathcal{C}(r, \gamma)$, and let $\beta$ be defined in terms of $\alpha$ by (C.30), where no eigenvalues of the matrix A lie on the imaginary axis. Then $\beta \in C^{r}\left(\mathbb{T}^{n}, \mathbb{R}^{m}\right)$, and there exists a uniform constant $C>0$ such that $\|\beta\|_{C^{r}} \leq C\|\alpha\|_{\mathcal{C}(r, \gamma)}$.

Proof. We treat the case where all eigenvalues of $A$ have negative real parts. The more general case where the eigenvalues of $A$ have nonzero real parts may be handled by using the decomposition of $\mathbb{R}^{m}$ into $A$-invariant subspaces, $\mathbb{R}^{m}=E^{s} \oplus E^{u}$, as outlined in the proof of Theorem C.1 in the case of hypothesis (i).

Clearly $\beta \in C^{r-1}\left(\mathbb{T}^{n}, \mathbb{R}^{m}\right)$ and

$$
\left(D^{\mu} \beta\right)(\eta)=-\int_{0}^{\infty} e^{\tau A} D^{\mu} \alpha(\eta-\tau \gamma) d \tau
$$

for every $n$-dimensional multi-index $\mu$ with $|\mu| \leq r-1$. To show that $\beta \in C^{r}\left(\mathbb{T}^{n}, \mathbb{R}^{m}\right)$, it suffices to show that, for every vector $v \in \mathbb{R}^{n}$ with $|v|=1, d_{v} D^{\mu} \beta$ exists and is continuous. Let $v \in \mathbb{R}^{n}$ with $|v|=1$. Then there exists a constant $a$ with $|a| \leq 1$ and a vector $w \in \mathbb{R}^{n}$

Copyright (C) by SIAM. Unauthorized reproduction of this article is prohibited. 
with $|w| \leq 1$ such that $v=a \gamma+w$. Note that $d_{\gamma} D^{\mu} \beta$ exists and is continuous, since

$$
\begin{aligned}
d_{\gamma} D^{\mu} \beta(\eta) & =-d_{\gamma} \int_{0}^{\infty} e^{\tau A} D^{\mu} \alpha(\eta-\tau \gamma) d \tau \\
& =-\left.\frac{d}{d t}\right|_{t=0} \int_{0}^{\infty} e^{\tau A} D^{\mu} \alpha(\eta+(t-\tau) \gamma) d \tau \\
& =-\left.\frac{d}{d t}\right|_{t=0} e^{t A} \int_{-\infty}^{t} e^{-s A} D^{\mu} \alpha(\eta+s \gamma) d s \\
& =-A \int_{0}^{\infty} e^{\tau A} D^{\mu} \alpha(\eta-\tau \gamma) d \tau-D^{\mu} \alpha(\eta) .
\end{aligned}
$$

Also, $d_{w} D^{\mu} \beta$ exists and is continuous, since

$$
d_{w} D^{\mu} \beta(\eta)=-\int_{0}^{\infty} e^{\tau A} d_{w} D^{\mu} \alpha(\eta-\tau \gamma) d \tau .
$$

Then $d_{v} D^{\mu} \beta$ must exist and be continuous, since

$$
d_{v} D^{\mu} \beta=a d_{\gamma} D^{\mu} \beta+d_{w} D^{\mu} \beta .
$$

Therefore $\beta \in C^{r}\left(\mathbb{T}^{n}, \mathbb{R}^{m}\right)$.

Since $A$ has all eigenvalues with negative real parts, there exist positive constants $\lambda$ and $c$ such that $\left\|e^{\tau A}\right\| \leq c e^{-\lambda \tau}$ for all $\tau \geq 0$. We then have the following estimates:

$$
\begin{aligned}
\|\beta\|_{C^{r-1}} & \leq\left|\int_{0}^{\infty} e^{\tau A}\|\alpha\|_{C^{r-1}} d \tau\right| \\
& \leq\left|\int_{0}^{\infty} c e^{-\lambda \tau}\|\alpha\|_{C^{r-1}} d \tau\right| \\
& \leq \frac{c}{\lambda}\|\alpha\|_{C^{r-1}} \\
& \leq \frac{c}{\lambda}\|\alpha\|_{\mathcal{C}(r, \gamma)}
\end{aligned}
$$

and

$$
\begin{aligned}
\left\|d_{v} D^{\mu} \beta\right\|_{C^{0}} \leq & a\left\|d_{\gamma} D^{\mu} \beta\right\|_{C^{0}}+\left\|d_{w} D^{\mu} \beta\right\|_{C^{0}} \\
\leq & a\|A\| \int_{0}^{\infty} c e^{-\lambda \tau}\left\|D^{\mu} \alpha\right\|_{C^{0}} d \tau+a\left\|D^{\mu} \alpha\right\|_{C^{0}} \\
& +\int_{0}^{\infty} c e^{-\lambda \tau}\left\|d_{w} D^{\mu} \alpha\right\|_{C^{0}} \\
\leq & \left(\frac{a c\|A\|}{\lambda}+a+\frac{c}{\lambda}\right)\|\alpha\|_{\mathcal{C}(r, \gamma)} .
\end{aligned}
$$

Thus we have the following estimate on the $C^{r}$ norm of $\beta$ :

$$
\begin{aligned}
\|\beta\|_{C^{r}} & \leq\|\beta\|_{C^{r-1}}+\sup _{|\mu|=r-1,|v|=1}\left\|d_{v} D^{\mu} \beta\right\|_{C^{0}} \\
& \leq\left(\frac{a c\|A\|}{\lambda}+a+\frac{2 c}{\lambda}\right)\|\alpha\|_{\mathcal{C}(r, \gamma)} .
\end{aligned}
$$

Copyright (C) by SIAM. Unauthorized reproduction of this article is prohibited. 
Proposition C.4. Suppose that the vector $\nu \gamma \in \mathbb{Q}^{n}$, let $\alpha \in \mathcal{C}(r, \gamma)$, and let $\beta$ be defined in terms of $\alpha$ by (C.22), where the matrix $A$ has no eigenvalues of the form $i N q_{2} /\left(\nu q_{1}\right)$ for an integer $N$, where $q_{1}$ is the least common denominator of the absolute values of all the components of $\nu \gamma$, and $q_{2}$ is the greatest common divisor of all of the components of $q_{1} \nu \gamma$. Then $\beta \in C^{r}\left(\mathbb{T}^{n}, \mathbb{R}^{m}\right)$, and there exists a uniform constant $C>0$ such that $\|\beta\|_{C^{r}} \leq C\|\alpha\|_{\mathcal{C}(r, \gamma)}$.

Proof. The proof is similar to the proof of Proposition C.3.

Theorem C.5. Suppose that system (C.1) is $C^{1}$, that $f(0)=0$, and that all the eigenvalues of the matrix $A:=D f(0)$ have negative real parts. Then for sufficiently small $\epsilon>0$ system (C.1) has an attracting invariant torus of dimension $n+1$, which is a continuation from the trivial manifold $\{0\} \times \mathbb{T}^{n} \times \mathbb{T}^{1}$ at $\epsilon=0$.

Proof. By Theorem C.1 under hypothesis (i), there exists $\beta \in C^{r}\left(\mathbb{T}^{n}, \mathbb{R}^{m}\right)$, depending smoothly on $\epsilon$, such that the $P$-invariant manifold $\{0\} \times \mathbb{T}^{n}$ at $\epsilon=0$ continues to the $P$ invariant manifold $\mathcal{M}_{\epsilon}:=\left\{(\beta(\eta), \eta): \eta \in \mathbb{T}^{n}\right\}$ for $\epsilon>0$ sufficiently small, where $P$ is the Poincaré map defined in (C.3). In particular, since $\mathcal{M}_{\epsilon}$ is at least Lipschitz continuous in $\epsilon$, there exist positive constants $\epsilon_{0}$ and $M$ such that

$$
\|\beta\|_{C^{0}} \leq M \epsilon
$$

for all $\epsilon \in\left(0, \epsilon_{0}\right)$.

It remains to show attraction. By a theorem in [13], it suffices to show that

$$
\sup _{\eta \in \mathbb{T}^{n}}\|\Pi D P(\beta(\eta), \eta, \epsilon)\|<1
$$

where $\Pi$ is the projection onto the normal component of the manifold $\mathcal{M}_{\epsilon}$ and $\|\cdot\|$ denotes the usual operator norm.

From (C.4), we have that

$$
\Pi D P(\beta(\eta), \eta, \epsilon)=I+\epsilon^{k} \frac{2 \pi}{\omega(\beta(\eta), \eta)} D f(\beta(\eta))+O\left(\epsilon^{k+1}\right),
$$

where $I$ denotes the $m \times m$ identity matrix. Expanding about $J=0$ and using (C.42), we obtain

$$
\Pi D P(\beta(\eta), \eta, \epsilon)=I+\epsilon^{k} \frac{2 \pi}{\omega(0, \eta)} A+O\left(\epsilon^{k+1}\right) .
$$

Thus there exists a positive constant $b$ such that, for every $\eta \in \mathbb{T}^{n}$,

$$
\|\Pi D P(\beta(\eta), \eta, \epsilon)\| \leq\left\|I+\epsilon^{k} \frac{2 \pi}{\omega(0, \eta)} A\right\|+b \epsilon^{k+1}
$$

for $\epsilon$ sufficiently small.

Let $a$ be a positive constant such that every eigenvalue of $A$ has real part less than $-a$. By a lemma in [14], there exists a basis of $\mathbb{R}^{m}$ with a corresponding norm $|\cdot|_{H}$ and inner product $\langle\cdot, \cdot\rangle_{H}$ such that

$$
\langle A v, v\rangle_{H} \leq-a|v|_{H}^{2}
$$


for all $v \in \mathbb{R}^{m}$. This implies that

$$
\begin{aligned}
\left\|I+\epsilon^{k} \frac{2 \pi}{\omega(0, \eta)} A\right\|^{2} & =\sup _{|v|_{H}=1}\left|\left(I+\epsilon^{k} \frac{2 \pi}{\omega(0, \eta)} A\right) v\right|_{H}^{2} \\
& =\sup _{|v|_{H}=1}\left\langle\left(I+\epsilon^{k} \frac{2 \pi}{\omega(0, \eta)} A\right) v,\left(I+\epsilon^{k} \frac{2 \pi}{\omega(0, \eta)} A\right) v\right\rangle_{H} \\
& =\sup _{|v|_{H}=1}\left(|v|_{H}^{2}+\epsilon^{k} \frac{4 \pi}{\omega(0, \eta)}\langle A v, v\rangle_{H}+\epsilon^{2 k} \frac{4 \pi^{2}}{(\omega(0, \eta))^{2}}|A v|_{H}^{2}\right) \\
& \leq 1-\epsilon^{k} \frac{4 \pi}{\omega(0, \eta)} a+\epsilon^{2 k} \frac{4 \pi^{2}}{(\omega(0, \eta))^{2}}\|A\|^{2},
\end{aligned}
$$

and hence

$$
\left(\left\|I+\epsilon^{k} \frac{2 \pi}{\omega(0, \eta)} A\right\|+b \epsilon^{k+1}\right)^{2} \leq 1-\epsilon^{k} \frac{4 \pi a}{\omega(0, \eta)}+O\left(\epsilon^{k+1}\right),
$$

which implies that

$$
\left\|I+\epsilon^{k} \frac{2 \pi}{\omega(0, \eta)} A\right\|+b \epsilon^{k+1}<1
$$

for $\epsilon$ sufficiently small. Combining this with (C.46), we obtain (C.43) for $\epsilon>0$ sufficiently small. (Recall that $\omega(0, \eta)$ is bounded below by $\omega_{0}>0$ and is a continuous function of $\eta$ on the compact set $\mathbb{T}^{n}$.)

Thus $\mathcal{M}_{\epsilon}$ is an attracting invariant manifold for the Poincaré map $P$. Therefore $\mathcal{M}_{\epsilon} \times \mathbb{T}^{1}$ is an attracting invariant manifold for the ODE system (C.1), which is a continuation of the invariant manifold $\{0\} \times \mathbb{T}^{n} \times \mathbb{T}^{1}$ at $\epsilon=0$.

\section{REFERENCES}

[1] A. Blanchard, Phase-Locked Loops: Application to Coherent Receiver Design, Krieger, Malabar, FL, 1992.

[2] P. V. Brennan, Phase-Locked Loops, Principles and Practice, McGraw-Hill, New York, 1996.

[3] C. Chicone, Ordinary Differential Equations with Applications, 2nd ed., Springer-Verlag, New York, 2006.

[4] J. B. Encinas, Phase Locked Loops, Chapman \& Hall, New York, 1993.

[5] T. Endo And L. O. ChuA, Chaos from phase-locked loops, IEEE Trans. Circuits Systems, 35 (1988), pp. 987-1003.

[6] S. Engelberg And M. Wexler, The operation of the phase-lock loop, Comput. Math. Appl., 40 (2000), pp. 1253-1261.

[7] N. Fenichel, Persistence and smoothness of invariant manifolds for flows, Indiana U. Math. J., 21 (1971), pp. 193-226.

[8] F. M. Gardner, Phaselock Techniques, 3rd ed., Wiley, Hoboken, NJ, 2005.

[9] J. Guckenheimer And P. Holmes, Nonlinear Oscillations, Dynamical Systems, and Bifurcations of Vector Fields, Springer-Verlag, New York, 1983.

[10] J. Hale, Ordinary Differential Equations, 2nd ed., Krieger, Huntington, NY, 1980.

[11] V. F. Kroupa, Phase Lock Loops and Frequency Synthesis, Wiley, Hoboken, NJ, 2003.

Copyright (c) by SIAM. Unauthorized reproduction of this article is prohibited. 
[12] J. Kudrewicz And S. Wasowicz, Equations of Phase-Locked Loops: Dynamics on Circle, Torus and Cylinder, World Scientific, Singapore, 2007.

[13] M. Hirsch, C. Pugh, And M. Shub, Invariant Manifolds, Lecture Notes in Math. 583, Springer-Verlag, New York, 1977.

[14] M. Hirsch And S. Smale, Differential Equations, Dynamical Systems, and Linear Algebra, Academic Press, New York, 1974.

[15] W. C. Lindsey and C. M. Chie, Phase-Locked Loops, IEEE Press, New York, 1985.

[16] N. I. Margaris, Theory of the Non-linear Analog Phase Locked Loop, Springer, New York, 2004.

[17] National Semiconductor Corporation, DS007853, LM565/LM565C PLL data sheet, 1999; available online at, e.g., http://www.ti.com/lit/ds/snosbu1b/snosbu1b.pdf.

[18] E. Sanchez-Palencia, Methode de Centrage et Comportement des Trajectoires dans l'Espace des Phases, C. R. Acad. Sci. Paris Ser. A, 280 (1975), pp. 105-107.

[19] J. A. Sanders, F. Verhulst, And J. Murdock, Averaging Methods in Nonlinear Dynamical Systems, 2nd ed., Springer-Verlag, New York, 2007.

[20] J. A. SAnders, Asymptotic approximations and extension of time-scales, SIAM J. Math. Anal., 11 (1980), pp. $758-770$.

[21] J. L. Stensby, Phase-Locked Loops: Theory and Applications, CRC Press, Boca Raton, FL, 1997.

[22] S. Strogatz, Nonlinear Dynamics and Chaos, Perseus Books, New York, 1994.

Copyright $\odot$ by SIAM. Unauthorized reproduction of this article is prohibited. 